\title{
Four point functions in CFT's with slightly broken higher spin symmetry
}

\author{
Joao A. Silva \\ Fields and Strings Laboratory, Institute of Physics, \\ École Polytechnique Fédérale de Lausanne (EPFL), \\ CH-1015 Lausanne, Switzerland \\ E-mail: joao.alvesdasilva@epfl.ch
}

ABSTRACT: We compute spinning four point functions in the quasi-fermionic three dimensional conformal field theory with slightly broken higher spin symmetry at finite t'Hooft coupling. More concretely, we obtain a formula for $\left\langle j_{s} j_{\tilde{0}} j_{\tilde{0}} j_{\tilde{0}}\right\rangle$, where $j_{s}$ is a higher spin current and $j_{\tilde{0}}$ is the scalar single trace operator. Our procedure consists in writing a plausible ansatz in Mellin space and using crossing, pseudo-conservation and Regge boundedness to fix all undetermined coefficients. Our method can potentially be generalised to compute all spinning four point functions in these theories.

Keywords: Conformal Field Theory, Higher Spin Symmetry

ArXiv EPrint: 2103.00275 


\section{Contents}

1 Introduction and summary of results 1

2 The bootstrap of $\left\langle j_{s} j_{\tilde{0}} j_{\tilde{0}} j_{\tilde{0}}\right\rangle$

3 Bound on chaos for $\left\langle j_{s} j_{\tilde{0}} j_{\tilde{0}} j_{\tilde{0}}\right\rangle \quad 7$

3.1 Review of the bound on chaos and Rindler positivity 8

3.2 Consequences for $\left\langle j_{s} j_{\tilde{0}} j_{\tilde{0}} j_{\tilde{0}}\right\rangle_{c b} \quad 9$

3.3 The Regge limit of AdS contact diagrams for the parity even structure in $\left\langle j_{s} j_{\tilde{0}} j_{\tilde{0}} j_{\tilde{0}}\right\rangle$

3.4 The Regge limit of AdS contact diagrams for the parity odd structure in $\left\langle j_{s} j_{\tilde{0}} j_{\tilde{0}} j_{\tilde{0}}\right\rangle$

4 Open directions

A Bulk point limit

A.1 Bulk point singularity of an AdS contact diagram for a scalar four point function of unequal primaries

A.2 Bulk point singularity of AdS contact diagrams for $\left\langle j_{s} j_{\tilde{0}} j_{\tilde{0}} j_{\tilde{0}}\right\rangle$

A.3 Bulk point singularity of $\left\langle j_{s} j_{\tilde{0}} j_{\tilde{0}} j_{\tilde{0}}\right\rangle$ in CFT's with slightly broken higher spin symmetry

B Algorithm for computing $\left\langle j_{s} j_{\tilde{0}} j_{\tilde{0}} j_{\tilde{0}}\right\rangle$ in position space

C Mixed Fourier transform

D Miscellaneous formulas

E Contact interactions for scattering amplitudes

\section{Introduction and summary of results}

The dualities between conformal field theories and higher spin gravity theories in AdS are one of the most intriguing topics in the AdS/CFT correspondence. Potentially, these dualities should allow for an improved understanding of the AdS/CFT correspondence, since both sides of the duality are simple, at least when compared to the more standard case of $\mathcal{N}=4$ SYM and type IIB superstring theory. ${ }^{1}$ Of particular interest are CFT's with slightly broken higher spin symmetry, that were studied most notably in the paper by

\footnotetext{
${ }^{1}$ See [1] (which builds on the works [2-4]) for recent progress, where the path integral for critical $O(N)$ models was written in terms of higher spin gauge fields defined in the bulk of AdS.
} 
Maldacena and Zhiboedov [5], where all three point functions of single trace operators at the planar level were computed at finite t'Hooft coupling. In our paper, we compute some four point functions of spinning single trace operators at the planar level at finite t'Hooft coupling. The formulas we obtain are very simple and our formalism, which is based on pure CFT arguments in which Mellin space plays an important role, potentially paves the way for the computation of all spinning four point functions.

CFT's with slightly broken higher spin symmetry are large $N$ CFT's where higher spin symmetry is broken by $1 / N$ effects. There are two such theories, the quasi-boson theory and the quasi-fermion theory, which are defined in 3 dimensions. We will focus on the quasi-fermion theory. This theory depends on two parameters, $\tilde{N}$ and $\tilde{\lambda}$ (we follow the notation of [5]). We will study the theory at the planar level, i.e. at leading order in $\tilde{N}$. In that case the theory interpolates between the free fermion theory at $\tilde{\lambda}=0$ and the critical point of the $O(N)$ model (critical boson) at $\tilde{\lambda}=\infty$.

Being a large $N$ theory, the spectrum of the quasi-fermion theory organises into single and multitrace primary operators. Let us describe the single trace operators. There is one single trace operator for each even spin $s=0,2, \ldots$. The scalar primary, which we will denote by $j_{\tilde{0}}$, has dimension $2+O\left(\frac{1}{\tilde{N}}\right)[6]$. The spin 2 primary $j_{2}$ is exactly conserved. A higher spin primary $j_{s}$ of spin $s>2$ has dimension $s+1$ and acquires anomalous dimensions of $O\left(\frac{1}{\tilde{N}}\right)[7,8]$.

This theory is believed to be solvable in the planar limit. In [5] three point functions of single trace operators were computed at the planar level and for finite $\tilde{\lambda}$ through the use of slightly broken higher spin Ward identities. ${ }^{2}$ In [10] four point functions of scalar operators were computed using the Lorentzian inversion formula and Schwinger-Dyson equations. In [11] the four point function $\left\langle j_{2} j_{\tilde{0}} j_{\tilde{0}} j_{\tilde{0}}\right\rangle$ was computed using the pseudo-conservation equations. $^{3}$

We obtain a formula for $\left\langle j_{s} j_{\tilde{0}} j_{\tilde{0}} j_{\tilde{0}}\right\rangle$ for generic spin $s \geq 4$ :

$$
\left\langle j_{s} j_{\tilde{0}} j_{\tilde{0}} j_{\tilde{0}}\right\rangle=\frac{1}{\tilde{N} \sqrt{1+\tilde{\lambda}^{2}}}\left\langle j_{s} j_{\tilde{0}} j_{\tilde{0}} j_{\tilde{0}}\right\rangle_{f f}+\frac{\tilde{\lambda}}{\tilde{N} \sqrt{1+\tilde{\lambda}^{2}}}\left\langle j_{s} j_{\tilde{0}} j_{\tilde{0}} j_{\tilde{0}}\right\rangle_{c b}
$$

where $\left\langle j_{s} j_{\tilde{0}} j_{\tilde{0}} j_{\tilde{0}}\right\rangle_{f f}$ is the correlator in the free fermion theory (which is fully known) and $\left\langle j_{s} j_{\tilde{0}} j_{\tilde{0}} j_{\tilde{0}}\right\rangle_{c b}$ is the corresponding correlator in the critical boson theory. The critical boson theory is the IR fixed point of the theory of $\tilde{N}$ free real scalar fields perturbed by $\left(\phi_{i} \phi_{i}\right)^{2}$.

This result agrees with the $3 \mathrm{~d}$ bosonization picture advanced in [5], where it is proposed that the quasi-fermionic theory interpolates between a tridimensional theory of $\tilde{N}$ free fermions and the critical theory of $\tilde{N}$ bosons, in the limits $\tilde{\lambda} \rightarrow 0$ and $\tilde{\lambda} \rightarrow \infty$ respectively.

We obtain that

$$
\begin{aligned}
\left\langle j_{s} j_{\tilde{0}} j_{\tilde{0}} j_{\tilde{0}}\right\rangle_{c b}= & \left|x_{1}-x_{3}\right|^{-4 s-2}\left|x_{2}-x_{3}\right|^{2 s-1}\left|x_{2}-x_{4}\right|^{-2 s-3}\left|x_{3}-x_{4}\right|^{2 s-1} \\
& \times \sum_{k=0}^{s} \iint \frac{d \gamma_{12} d \gamma_{14}}{(2 \pi i)^{2}} M\left(\gamma_{12}, \gamma_{14} ; s, k\right) u^{-\gamma_{12}} v^{-\gamma_{14}} V(1 ; 2,3)^{s-k} V(1 ; 3,4)^{k},
\end{aligned}
$$

\footnotetext{
${ }^{2}$ This calculation was reproduced using higher spin techniques in [9], where also the parity odd structures were given.

${ }^{3}$ Correlators in ABJ theory were computed using slightly broken higher spin symmetry in [12].
} 
where $V(i ; j, k)$ is a conformal structure (see (2.3)) and $u$ and $v$ are the usual conformal cross ratios. $M\left(\gamma_{12}, \gamma_{14} ; s, k\right)$ is equal to

$$
\begin{aligned}
M\left(\gamma_{12}, \gamma_{14} ; s, k\right)= & \Gamma\left(-k+\gamma_{14}-1\right) \Gamma\left(-k+\gamma_{14}+\frac{1}{2}\right) \Gamma\left(s-\gamma_{12}-\gamma_{14}\right) \\
& \times \Gamma\left(s-\gamma_{12}-\gamma_{14}+\frac{3}{2}\right) \Gamma\left(k-s+\gamma_{12}-1\right) \\
& \times \Gamma\left(k-s+\gamma_{12}+\frac{1}{2}\right) p\left(\gamma_{12}, \gamma_{14} ; s, k\right)
\end{aligned}
$$

where $p\left(\gamma_{12}, \gamma_{14} ; s, k\right)$ is a polynomial in $\gamma_{12}$ and $\gamma_{14}$. This polynomial is fully determined by crossing, pseudo-conservation and Regge boundedness, see equations (2.8) and (2.9), see (2.11) and see also (3.16), (3.17) and (3.18).

We explain in section 2 how formula (1.1) solves the crossing and pseudo-conservation equations and correctly accounts for the exchange of single trace operators with the OPE coefficients derived in [5]. In section 3 we show that formula (1.1) is the unique solution to the pseudo-conservation and crossing equations which is consistent with the bound on chaos. In particular we analyse AdS contact diagrams for $\left\langle j_{s} j_{\tilde{0}} j_{\tilde{0}} j_{\tilde{0}}\right\rangle$ and we conclude that such diagrams violate the bound on chaos, provided $s \geq 4$. In section 4 we discuss open directions. In appendix A we study the bulk point limit of $\left\langle j_{s} j_{\tilde{0}} j_{\tilde{0}} j_{\tilde{0}}\right\rangle$. In appendix B we calculate $\left\langle j_{s} j_{\tilde{0}} j_{\tilde{0}} j_{\tilde{0}}\right\rangle$ in position space for spins $s=2, \ldots, 14$. This calculation agrees with the Mellin space result. In appendix C we recompute $\left\langle j_{2} j_{\tilde{0}} j_{\tilde{0}} j_{\tilde{0}}\right\rangle$ by solving the higher spin Ward identities.

\section{The bootstrap of $\left\langle j_{s} j_{\tilde{0}} j_{\tilde{0}} j_{\tilde{0}}\right\rangle$}

We will compute $\left\langle j_{s} j_{\tilde{0}} j_{\tilde{0}} j_{\tilde{0}}\right\rangle$. Let us start by examining the $\tilde{N}$ and $\tilde{\lambda}$ dependence. It is expected that the quasi-fermion theory interpolates between a theory of $\tilde{N}$ free fermions at $\tilde{\lambda}=0$ and the critical boson theory at $\tilde{\lambda}=\infty$.

We will work in a normalization where $\left\langle j_{s} j_{s}\right\rangle \sim 1$, i.e. two point functions of single trace operators do not depend on $\tilde{N}$ or $\tilde{\lambda}$. We use the $\sim$ sign to mean that we do not keep track of numerical factors, but we do keep track of the $\tilde{N}$ and $\tilde{\lambda}$ dependence. Thus, $\left\langle j_{s} j_{\tilde{0}} j_{\tilde{0}} j_{\tilde{0}}\right\rangle \sim \frac{1}{\tilde{N}}$. At this order, we can only have exchanges of single trace operators or double trace operators $\left[j_{\tilde{0}}, j_{\tilde{0}}\right]$ or $\left[j_{s}, j_{\tilde{0}}\right]$.

Let us consider exchanges of single trace operators. The relevant three point functions are $\left\langle j_{s} j_{\tilde{0}} j_{s^{\prime}}\right\rangle$ and $\left\langle j_{s^{\prime}} j_{\tilde{0}} j_{\tilde{0}}\right\rangle$, with $s^{\prime} \geq 2$. Note that $\left\langle j_{\tilde{0}} j_{\tilde{0}} j_{\tilde{0}}\right\rangle=0$ [5]. From [5] we see that $\left\langle j_{s} j_{0} j_{0}\right\rangle \sim \frac{1}{\sqrt{\tilde{N}}}$. There are two possible structures for $\left\langle j_{s} j_{\tilde{0}} j_{s^{\prime}}\right\rangle$, the fermion and the odd structure. We have that $\left\langle j_{s} j_{\tilde{0}} j_{s^{\prime}}\right\rangle_{\text {fermion }} \sim \frac{1}{\sqrt{\tilde{N}} \sqrt{1+\tilde{\lambda}^{2}}}$ and $\left\langle j_{s} j_{\tilde{0}} j_{s^{\prime}}\right\rangle_{\text {odd }} \sim \frac{\tilde{\lambda}}{\sqrt{\tilde{N}} \sqrt{1+\tilde{\lambda}^{2}}}$.

Based on this we propose the following ansatz

$$
\left\langle j_{s} j_{\tilde{0}} j_{\tilde{0}} j_{\tilde{0}}\right\rangle=\frac{1}{\tilde{N} \sqrt{1+\tilde{\lambda}^{2}}}\left\langle j_{s} j_{\tilde{0}} j_{\tilde{0}} j_{\tilde{0}}\right\rangle_{f f}+\frac{\tilde{\lambda}}{\tilde{N} \sqrt{1+\tilde{\lambda}^{2}}}\left\langle j_{s} j_{\tilde{0}} j_{\tilde{0}} j_{\tilde{0}}\right\rangle_{c b}
$$

where $\left\langle j_{s} j_{\tilde{0}} j_{\tilde{0}} j_{\tilde{0}}\right\rangle_{f f}$ is the four point function in the free fermion theory, whose form can be read in [13]. To the best of our knowledge, $\left\langle j_{s} j_{\tilde{0}} j_{\tilde{0}} j_{\tilde{0}}\right\rangle_{c b}$ has not yet been computed and it 
will be the subject of this section to do precisely that. We attached the subscript $c b$ since it is expected that it corresponds to a four point function in the critical boson theory.

The reader might be confused about the factor of $\frac{1}{\tilde{N}}$. In our normalization, $\lim _{\tilde{\lambda} \rightarrow 0}\left\langle j_{s} j_{\tilde{0}} j_{\tilde{0}} j_{\tilde{0}}\right\rangle=\frac{\left\langle j_{s} j_{\tilde{0}} j_{\tilde{0}} j_{\tilde{0}}\right\rangle_{f f}}{\hat{N}}$ and $\lim _{\tilde{\lambda} \rightarrow \infty}\left\langle j_{s} j_{\tilde{0}} j_{\tilde{0}} j_{\tilde{0}}\right\rangle=\frac{\left\langle j_{s} j_{\tilde{0}} j_{\tilde{0}} j_{\tilde{0}}\right\rangle_{c b}}{\hat{N}}$. Given that the quasifermionic theory interpolates between a theory of $\tilde{N}$ free fermions and the critical theory of $\tilde{N}$ bosons, the reader might be confused about why there is a factor of $\frac{1}{N}$. There are two things happening in this context. First, when we write $\left\langle j_{s} j_{\tilde{0}} j_{\tilde{0}} j_{\tilde{0}}\right\rangle_{f f}$ and $\left\langle j_{s} j_{\tilde{0}} j_{\tilde{0}} j_{\tilde{0}}\right\rangle_{c b}$ we have decided to factor out the dependence on $\tilde{N}$. Second, the reader might thus have expected to encounter $\left\langle j_{s} j_{\tilde{0}} j_{\tilde{0}} j_{\tilde{0}}\right\rangle \sim \tilde{N}$, but this is only true when two point functions are normalized such that $\left\langle j_{s} j_{s}\right\rangle \sim\left\langle j_{\tilde{0}} j_{\tilde{0}}\right\rangle \sim \tilde{N}$, whereas we are using different normalizations, namely $\left\langle j_{s} j_{s}\right\rangle \sim\left\langle j_{\tilde{0}} j_{\tilde{0}}\right\rangle \sim 1$. This justifies why does $\left\langle j_{s} j_{\tilde{0}} j_{\tilde{0}} j_{\tilde{0}}\right\rangle \sim \frac{1}{\tilde{N}}$.

We can write parity even and parity odd structures for the correlator $\left\langle j_{s} j_{\tilde{0}} j_{\tilde{0}} j_{\tilde{0}}\right\rangle$. The parity odd structures are realised in the free fermion theory. This is because $j_{\tilde{0}}$ is parity odd in the free fermion theory. The parity even structures are realised in the quasi-boson theory. Thus, we write

$$
\left\langle j_{s} j_{\tilde{0}} j_{\tilde{0}} j_{\tilde{0}}\right\rangle_{c b}=\sum_{k=0}^{s} f_{k}\left(x_{i j}\right) V(1 ; 2,3)^{s-k} V(1 ; 3,4)^{k},
$$

where $V(i ; j, k)$ is a conformal structure which is given in embedding space [14] by

$$
V(i ; j, k)=\frac{\left(Z_{i} \cdot P_{j}\right)\left(P_{i} \cdot P_{k}\right)-\left(Z_{i} \cdot P_{k}\right)\left(P_{i} \cdot P_{j}\right)}{P_{j} \cdot P_{k}} .
$$

$P_{i}$ and $Z_{i}$ are null vectors on $\mathbb{R}^{3,2} . Z_{i}$ encodes the spinning indices. $f_{k}\left(x_{i j}\right)$ is a function of the distances between the points, with appropriate weights on each of the points. We find it advantageous to consider the Mellin representation

$$
\begin{aligned}
f_{k}\left(x_{i j}\right) & =\int\left[\frac{d \gamma_{i j}}{2 \pi i}\right] \hat{M}\left(\gamma_{i j} ; s, k\right) x_{i j}^{-2 \gamma_{i j}}, \\
\sum_{j \neq 1} \gamma_{1 j} & =2 s+1, \quad \sum_{j \neq i} \gamma_{i j}=2, \quad i=2,3,4 .
\end{aligned}
$$

Eq. (2.2) can be rewritten as

$$
\begin{aligned}
\left\langle j_{s} j_{\tilde{0}} j_{\tilde{0}} j_{\tilde{0}}\right\rangle_{c b}= & \left|x_{1}-x_{3}\right|^{-4 s-2}\left|x_{2}-x_{3}\right|^{2 s-1}\left|x_{2}-x_{4}\right|^{-2 s-3}\left|x_{3}-x_{4}\right|^{2 s-1} \\
& \times \sum_{k=0}^{s} \iint \frac{d \gamma_{12} d \gamma_{14}}{(2 \pi i)^{2}} \hat{M}\left(\gamma_{12}, \gamma_{14} ; s, k\right) u^{-\gamma_{12}} v^{-\gamma_{14}} V(1 ; 2,3)^{s-k} V(1 ; 3,4)^{k}
\end{aligned}
$$

We will call $\hat{M}\left(\gamma_{12}, \gamma_{14} ; s, k\right)$ the Mellin amplitude. ${ }^{4}$

\footnotetext{
${ }^{4}$ Spinning Mellin amplitudes are analysed in [15-17]. The definitions slightly differ among these works, but at least concerning conformal four point functions the basic idea is to decompose the correlator in a basis of spinning structures and take the Mellin transform with respect to each function of the positions multiplying each structure. Up to now all works use the embedding space formalism, which has the serious drawback of involving many degeneracies for arbitrary spinning correlators. For generic spinning correlators, we think it would be interesting to define Mellin amplitudes with the conformal frame formalism [18, 19], which does not have the problem of degeneracies. We think that it is an interesting problem to work out the poles and residues of the Mellin amplitude for spinning correlators using the conformal frame formalism.
} 
The location of the poles of the Mellin amplitude is related to the operator product expansion of the external operators. Let us make this point explicitly. Consider two external operators $O_{1}, O_{2}$ of dimensions $\Delta_{1}, \Delta_{2}$ and spins $s_{1}, s_{2}$ and suppose they exchange an operator of dimension $\Delta$ and spin $s$. Then the most singular term in the lightcone OPE is

$$
\mathcal{O}_{\mu_{1} \ldots \mu_{s_{1}}}(x) \mathcal{O}_{\nu_{1} \ldots \nu_{s_{2}}}(0) \supset \frac{\mathcal{O}_{\rho_{1} \ldots \rho_{s}}(0) x^{\rho_{1}} \ldots x^{\rho_{s}}}{\left(x^{2}\right)^{\frac{\Delta_{1}+\Delta_{2}+s_{1}+s_{2}}{2}-\frac{\tau}{2}}} x_{\left\{\mu_{1} \ldots \mu_{s_{1}}\right\}} x_{\left\{\nu_{1} \ldots \nu_{s_{2}}\right\}}\left(1+O\left(x^{2}\right)\right),
$$

where $\tau=\Delta-s$. From this logic we expect the Mellin amplitude to have poles at $\gamma_{12}=\frac{\Delta_{1}+\Delta_{2}+s_{1}+s_{2}}{2}-\frac{\tau}{2}-n$, where $n$ is a nonnegative integer.

For $\left\langle j_{s} j_{\tilde{0}} j_{\tilde{0}} j_{\tilde{0}}\right\rangle$ all OPE channels are equal. To order $\frac{1}{N}$ there can be exchanges of higher spin currents and double traces $\left[j_{s}, j_{\tilde{0}}\right]$ and $\left[j_{\tilde{0}}, j_{\tilde{0}}\right]$, which have twist 1,3 and 4 respectively. This motivates the following ansatz

$$
\begin{aligned}
\hat{M}\left(\gamma_{12}, \gamma_{14} ; s, k\right)= & \Gamma\left(-k+\gamma_{14}-1\right) \Gamma\left(-k+\gamma_{14}+\frac{1}{2}\right) \Gamma\left(-s+\gamma_{13}-1\right) \\
& \times \Gamma\left(-s+\gamma_{13}+\frac{1}{2}\right) \Gamma\left(k-s+\gamma_{12}-1\right) \\
& \times \Gamma\left(k-s+\gamma_{12}+\frac{1}{2}\right) p\left(\gamma_{12}, \gamma_{14} ; s, k\right),
\end{aligned}
$$

where $\gamma_{13}=2 s+1-\gamma_{12}-\gamma_{14}$. The $\Gamma$ functions contain all the poles implied by the OPE. For this reason we assume that $p\left(\gamma_{12}, \gamma_{14} ; s, k\right)$ is a polynomial in the Mellin variables.

The bound on chaos [20] bounds the degree of the polynomial $p\left(\gamma_{12}, \gamma_{14} ; s, k\right)$. This is worked out in section 3, see (3.16), (3.17) and (3.18) for the precise formulas. Furthermore, $\left\langle j_{s} j_{\tilde{0}} j_{\tilde{0}} j_{\tilde{0}}\right\rangle$ is constrained by invariance under interchange of points $2 \leftrightarrow 3$ and $2 \leftrightarrow 4$. This crossing symmetry implies the equations

$$
\begin{aligned}
& p\left(\gamma_{12}, \gamma_{14} ; s, k\right)=\sum_{k_{2}=k}^{s}(-1)^{k_{2}}\left(\begin{array}{c}
k_{2} \\
k
\end{array}\right) p\left(2 s+1-k_{2}-\gamma_{12}-\gamma_{14}, \gamma_{14}-k+k_{2} ; s, k_{2}\right), \\
& p\left(\gamma_{12}, \gamma_{14} ; s, k\right)=p\left(\gamma_{14}, \gamma_{12} ; s, s-k\right) .
\end{aligned}
$$

$\left\langle j_{s} j_{\tilde{0}} j_{\tilde{0}} j_{\tilde{0}}\right\rangle$ is constrained by pseudoconservation of $j_{s}$. We implement this condition in embedding space. The differential operator for conservation is $\frac{\partial}{\partial P_{1}^{A}} D_{A}$, where

$$
D_{A}=\left(\frac{d}{2}-1+Z_{1} \cdot \frac{\partial}{\partial Z_{1}}\right) \frac{\partial}{\partial Z_{1}^{A}}-\frac{1}{2}\left(Z_{1}\right)_{A} \frac{\partial^{2}}{\partial Z_{1} \cdot \partial Z_{1}} .
$$

Since $\partial \cdot j_{s}$ is a primary operator of spin $s-1$ and dimension $s+2$, then $\left\langle\partial \cdot j_{s} j_{\tilde{0}} j_{\tilde{0}} j_{\tilde{0}}\right\rangle$ is a conformal four point function of primary operators. $\left\langle\partial \cdot j_{s} j_{\tilde{0}} j_{\tilde{0}} j_{\tilde{0}}\right\rangle$ factorizes into products of a two point function times a three point function. Such a four point function is made up of powers of $u$ and of $v$ and so its Mellin amplitude vanishes.

Four point functions of scalars with vanishing Mellin amplitudes were analysed in [21], see in particular section E.E.1. A similar analysis can be performed for the spinning case, though we will not pursue it here. The important conclusion is that in Mellin space pseudoconservation is the same as conservation. In other words, $\left\langle\partial \cdot j_{s} j_{\tilde{0}} j_{\tilde{0}} j_{\tilde{0}}\right\rangle$ has a vanishing Mellin amplitude. 
Pseudoconservation implies the equation

$$
\sum_{i_{1}=-1}^{1} \sum_{i_{2}=-1}^{1} \sum_{i_{3}=-1}^{2} a_{i_{1}, i_{2}, i_{3}}\left(\gamma_{12}, \gamma_{14}\right) p\left(\gamma_{12}+i_{1}, \gamma_{14}+i_{2} ; s, k+i_{3}\right)=0 .
$$

The coefficients are written in the appendix D, see formula (D.1).

The crossing equations (2.8), (2.9), the pseudoconservation equation (2.11) and Regge boundedness (3.16), (3.17) and (3.18) determine $p\left(\gamma_{12}, \gamma_{14} ; s, k\right)$ up to a multiplicative constant. This has to do with the fact that we have not picked a normalization for the higher spin current $j_{s}$. It is simple to solve this set of equations in a computer algebra system for each spin $s$. We find that the solution always has the form

$$
\begin{array}{ll}
p\left(\gamma_{12}, \gamma_{14} ; s, k\right)=\sum_{k_{1}=0}^{k} \sum_{k_{2}=0}^{s-k} b\left(s, k ; k_{1}, k_{2}\right) \gamma_{12}^{k_{2}} \gamma_{14}^{k_{1}}, & k \leq \frac{s}{2} \\
p\left(\gamma_{12}, \gamma_{14} ; s, k\right)=p\left(\gamma_{14}, \gamma_{12} ; s, s-k\right), & k>\frac{s}{2}
\end{array}
$$

$p\left(\gamma_{12}, \gamma_{14} ; s, k\right)$ turns out to have degree $s$. Using a laptop we generated solutions up to spin 40. Picking a normalization in which $p\left(\gamma_{12}, \gamma_{14} ; s, k=0\right) \supset 1$, we find as an example that for $s=4$ we have

$$
\begin{aligned}
p\left(\gamma_{12}, \gamma_{14} ; s=4, k=0\right)= & 1-\frac{19 \gamma_{12}}{20}+\frac{119 \gamma_{12}^{2}}{360}-\frac{\gamma_{12}^{3}}{20}+\frac{\gamma_{12}^{4}}{360}, \\
p\left(\gamma_{12}, \gamma_{14} ; s=4, k=1\right)= & -\frac{8}{15}+\frac{4 \gamma_{12}}{9}-\frac{11 \gamma_{12}^{2}}{90}+\frac{\gamma_{12}^{3}}{90}+\left(\frac{2}{5}-\frac{11 \gamma_{12}}{30}+\frac{\gamma_{12}^{2}}{9}-\frac{\gamma_{12}^{3}}{90}\right) \gamma_{14} \\
p\left(\gamma_{12}, \gamma_{14} ; s=4, k=2\right)= & \frac{1}{5}-\frac{4 \gamma_{12}}{15}+\frac{\gamma_{12}^{2}}{15}+\left(-\frac{4}{15}+\frac{11 \gamma_{12}}{36}-\frac{13 \gamma_{12}^{2}}{180}\right) \gamma_{14} \\
& +\left(\frac{1}{15}-\frac{13 \gamma_{12}}{180}+\frac{\gamma_{12}^{2}}{60}\right) \gamma_{14}^{2}, \\
p\left(\gamma_{12}, \gamma_{14} ; s=4, k=3\right)= & -\frac{8}{15}+\frac{4 \gamma_{14}}{9}-\frac{11 \gamma_{14}^{2}}{90}+\frac{\gamma_{14}^{3}}{90}+\left(\frac{2}{5}-\frac{11 \gamma_{14}}{30}+\frac{\gamma_{14}^{2}}{9}-\frac{\gamma_{14}^{3}}{90}\right) \gamma_{12}, \\
p\left(\gamma_{12}, \gamma_{14} ; s=4, k=4\right)= & 1-\frac{19 \gamma_{14}}{20}+\frac{119 \gamma_{14}^{2}}{360}-\frac{\gamma_{14}^{3}}{20}+\frac{\gamma_{14}^{4}}{360}
\end{aligned}
$$

We write this correlator in position space in appendix D, see formula (D.2).

In appendix B we implement an algorithm to compute $\left\langle j_{s} j_{\tilde{0}} j_{\tilde{0}} j_{\tilde{0}}\right\rangle$ in position space. We managed to determine $\left\langle j_{s} j_{\tilde{0}} j_{\tilde{0}} j_{\tilde{0}}\right\rangle$ in position space for spins $2, \ldots, 14$ using this algorithm. We write the formulas for the correlators in position space in an accompanying notebook. Taking the Mellin transform we get precisely the same as we get with the procedure in Mellin space. The advantage of Mellin space is that it allows to write equations (2.8), (2.9) and (2.11) that determine the solution for generic $s$.

Let us mention some checks on our solution. One such check is compatibility of the pseudo-conservation equations with conformal symmetry. $\partial \cdot j_{s}$ is a conformal primary at leading order in $\frac{1}{\tilde{N}}$. $\partial \cdot j_{s}$ can have contributions coming from $\left[j_{s_{1}}, j_{\tilde{0}}\right]$ and $\left[j_{s_{1}}, j_{s_{2}}\right]$. Only 
the former matter since we are interested in $\left\langle j_{s} j_{\tilde{0}} j_{\tilde{0}} j_{\tilde{0}}\right\rangle$. More precisely,

$$
\partial \cdot j_{s} \supset \sum_{s_{1}=2}^{s-2} \sum_{m=0}^{s-s_{1}-1} c_{m} \partial^{m} j_{s_{1}} \partial^{s-s_{1}-1-m} j_{\tilde{0}} .
$$

The coefficients $c_{m}$ are fixed by conformal symmetry (see formula (B.7)). When we run our algorithm in position space we do not need to input the values of $c_{m}$, we prefer to keep them unknown. It turns out that our algorithm fixes $c_{m}$ in agreement with (B.7). This is an important check on our results.

We also checked that the short distance limit of our expression for $\left\langle j_{s} j_{\tilde{0}} j_{\tilde{0}} j_{\tilde{0}}\right\rangle_{c b}$ agrees with the correct three point structures for the exchange of higher spin currents. Let us take $s=4$ for concreteness. The short distance limit $u \rightarrow 0$ captures the exchange of the higher spin currents in the s-channel. If afterwards we take $v \rightarrow 1$, we find that the correlator behaves as

$$
\lim _{v \rightarrow 1} \lim _{u \rightarrow 0}\left\langle j_{s} j_{\tilde{0}} j_{\tilde{0}} j_{\tilde{0}}\right\rangle_{c b} \sim \sum_{J=2}^{\infty} \frac{1}{u^{5}} \frac{x_{34}^{7}}{x_{13}^{7} x_{14}^{11} x_{23}^{4}}(1-v)^{J} V(1 ; 2,3)^{4}
$$

The $\sim$ sign means that we just keep track of the conformal structure that appears, but we do not keep track of numerical coefficients. Eq. (2.16) is matched by the behaviour of conformal blocks of higher spin currents in the same limit.

Formula (1.1) correctly accounts for the exchange of single trace operators in $\left\langle j_{s} j_{\tilde{0}} j_{\tilde{0}} j_{\tilde{0}}\right\rangle$. However, it is not obvious that it correctly accounts for the exchange of double trace operators. Indeed, one can imagine adding to (1.1) AdS contact diagrams, which are solutions to crossing that only involve the exchange of double trace operators. By taking linear combinations of AdS contact diagrams one can furthermore obtain solutions to the conservation equations. However, in the next section we consider such linear combinations and show that they always violate the bound on chaos. For this reason, it is not legal to add them to (1.1).

\section{Bound on chaos for $\left\langle j_{s} j_{\tilde{0}} j_{\tilde{0}} j_{\tilde{0}}\right\rangle$}

The bound on chaos [22] constrains the Regge limit of $\left\langle j_{s} j_{\tilde{0}} j_{\tilde{0}} j_{\tilde{0}}\right\rangle$. In this section we review the bound on chaos and derive its consequences for $\left\langle j_{s} j_{\tilde{0}} j_{\tilde{0}} j_{\tilde{0}}\right\rangle$. There are two possible structures one can write for $\left\langle j_{s} j_{\tilde{0}} j_{\tilde{0}} j_{\tilde{0}}\right\rangle$. One structure involves the $\epsilon$ tensor and the other one does not. We examine the two cases separately in sections (3.2) and (3.4) and derive bounds on the Regge growth of the Mellin amplitude for both of these cases.

Solutions to crossing that only involve the exchange of double twist operators are given by AdS contact diagrams. This was proven in [23], for the special case of four point functions of external scalars. We will assume that such a result holds for any n-point function of spinning conformal primaries. We study AdS contact diagrams in sections (3.3) and (3.4). Our main conclusion is that AdS contact diagrams for $\left\langle j_{s} j_{\tilde{0}} j_{\tilde{0}} j_{\tilde{0}}\right\rangle$ are incompatible with the bound on chaos, provided $s \geq 4$. For $s=2$ we construct the contact diagrams that are compatible with the bound on chaos, see formulas (3.37) and (3.52). This completes the proof of formula (1.1). 


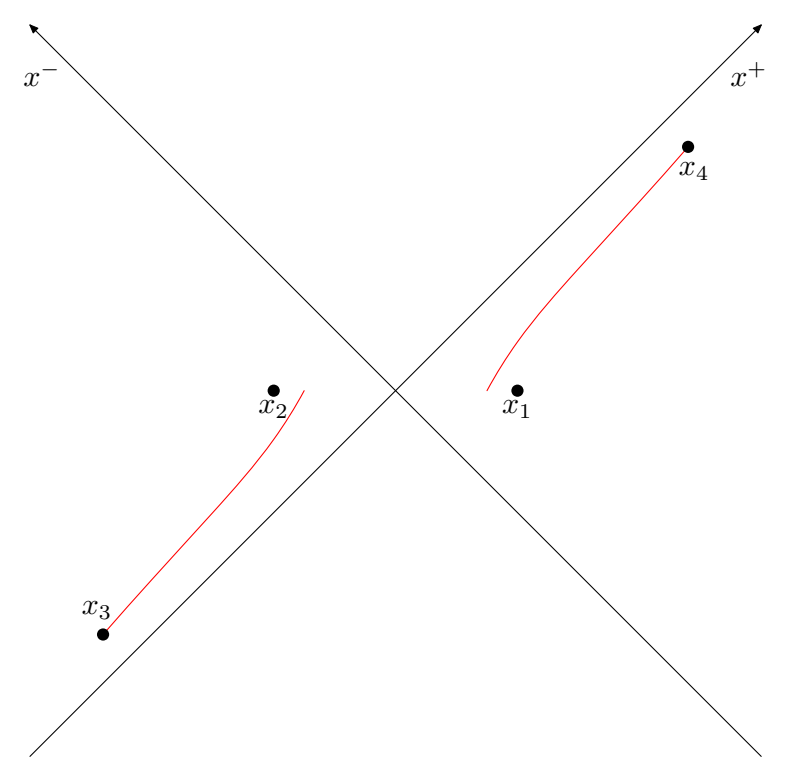

Figure 1. The Regge limit corresponds to taking $t \rightarrow \infty$ in (3.1).

\subsection{Review of the bound on chaos and Rindler positivity}

Conformal field theories are constrained by the Regge behaviour of Lorentzian correlators. For nonperturbative CFT's, correlators in the Regge limit are bounded by the Euclidean OPE in the first sheet. For large N CFT's one needs to use the bound on chaos to bound correlators in the Regge limit. In this subsection we review the bound on chaos [22].

We will consider the following kinematics for a four point function, in which we set all four points on the same plane $\left(x^{ \pm}=t \pm x\right)$

$$
x_{1}^{ \pm}= \pm 1, x_{2}^{ \pm}=\mp 1, x_{3}^{ \pm}=\mp e^{\rho \pm t}, x_{4}^{ \pm}= \pm e^{\rho \pm t},
$$

see figure 1.

The bound on chaos applies for systems at finite temperature with a large number of degrees of freedom. For the case of a large $\mathrm{N}$ conformal field theory, a correlation function of single trace primaries $\left\langle V\left(x_{1}\right) V\left(x_{2}\right) W\left(x_{3}\right) W\left(x_{4}\right)\right\rangle$ obeys

$$
\left\langle V\left(x_{1}\right) V\left(x_{2}\right) W\left(x_{3}\right) W\left(x_{4}\right)\right\rangle \approx\left\langle V\left(x_{1}\right) V\left(x_{2}\right)\right\rangle\left\langle W\left(x_{3}\right) W\left(x_{4}\right)\right\rangle\left(1+\alpha \frac{e^{\lambda_{L} t}}{N}\right),
$$

where the Lyapunov exponent $\lambda_{L}$ obeys the bound $\lambda_{L} \leq 2 \pi T$, where $T$ is the temperature of the system. The proportionality constant $\alpha$ does not depend on $t$. The bound on chaos can be applied to large N CFT's in Minkowski space, in which case we should consider the temperature $T=\frac{1}{2 \pi}$ of the Rindler horizon.

We cannot apply directly (3.2) to $\left\langle j_{s} j_{\tilde{0}} j_{\tilde{0}} j_{\tilde{0}}\right\rangle$. However, we can use Rindler positivity [24] to bound $\left\langle j_{s} j_{\tilde{0}} j_{\tilde{0}} j_{\tilde{0}}\right\rangle$ by $\left\langle j_{s} j_{s} j_{\tilde{0}} j_{\tilde{0}}\right\rangle$ and $\left\langle j_{\tilde{0}} j_{\tilde{0}} j_{\tilde{0}} j_{\tilde{0}}\right\rangle$ and use the bound on chaos to bound the latter two quantities, as we will explain next.

The Rindler conjugate $\bar{O}$ of an operator $O$ is defined as $\bar{O}_{\mu, \nu \ldots}(t, x, \vec{y})=$ $O_{\mu, \nu, \ldots}^{\dagger}(-t,-x, \vec{y})$, where $\vec{y}$ refers to a transverse coordinate relative to the plane of figure 1. Furthermore we have that $\overline{O_{1} O_{2}}=\bar{O}_{1} \bar{O}_{2}$. Rindler positivity and Cauchy-Schwarz 
inequalities imply that

$$
|\langle\bar{A} B\rangle|^{2} \leq\langle\bar{A} A\rangle\langle\bar{B} B\rangle
$$

where $A$ and $B$ are operators (that might be composite) defined on a single Rindler wedge.

Let us define $A=j_{\tilde{0}}\left(x_{3}\right) j_{s}\left(x_{2}\right), B=j_{\tilde{0}}\left(x_{2}\right) j_{\tilde{0}}\left(x_{3}\right)$. Then, the time-ordered correlation function in the configuration (3.1) is given by

$$
\begin{array}{r}
\left\langle T\left[j_{s}\left(x_{1}\right) j_{\tilde{0}}\left(x_{2}\right) j_{\tilde{0}}\left(x_{3}\right) j_{\tilde{0}}\left(x_{4}\right)\right]\right\rangle=\langle\bar{A} B\rangle \\
\leq \sqrt{\left\langle j_{\tilde{0}}\left(x_{4}\right) j_{s}\left(x_{1}\right) j_{\tilde{0}}\left(x_{3}\right) j_{s}\left(x_{2}\right)\right\rangle \times\left\langle j_{\tilde{0}}\left(x_{1}\right) j_{\tilde{0}}\left(x_{4}\right) j_{\tilde{0}}\left(x_{2}\right) j_{\tilde{0}}\left(x_{3}\right)\right\rangle}
\end{array}
$$

The bound on chaos on the r.h.s. of the previous expression implies a bound on $\left\langle j_{s} j_{\tilde{0}} j_{\tilde{0}} j_{\tilde{0}}\right\rangle$. In terms of $\sigma=e^{-t}$ :

$$
\lim _{t \rightarrow \infty}\left\langle T\left[j_{s}\left(x_{1}\right) j_{\tilde{0}}\left(x_{2}\right) j_{\tilde{0}}\left(x_{3}\right) j_{\tilde{0}}\left(x_{4}\right)\right]\right\rangle \sim \frac{\sigma^{\lambda_{1}}}{N}+O\left(\frac{1}{N^{2}}\right),
$$

where $\lambda_{1} \geq-1$.

\subsection{Consequences for $\left\langle j_{s} j_{\tilde{0}} j_{\tilde{0}} j_{\tilde{0}}\right\rangle_{c b}$}

Let us work out the consequences of the bound on chaos for the Mellin amplitudes of $\left\langle j_{s} j_{\tilde{0}} j_{\tilde{0}} j_{\tilde{0}}\right\rangle$. In the critical boson theory,

$$
\begin{aligned}
& \left\langle j_{s}\left(x_{1}\right) j_{\tilde{0}}\left(x_{2}\right) j_{\tilde{0}}\left(x_{3}\right) j_{\tilde{0}}\left(x_{4}\right)\right\rangle_{c b} \\
& =\left|x_{1}-x_{3}\right|^{-4 s-2}\left|x_{2}-x_{3}\right|^{2 s-1}\left|x_{2}-x_{4}\right|^{-2 s-3}\left|x_{3}-x_{4}\right|^{2 s-1} \\
& \quad \times \sum_{k=0}^{s} \iint \frac{d \gamma_{12} d \gamma_{14}}{(2 \pi i)^{2}} \hat{M}\left(\gamma_{12}, \gamma_{14} ; s, k\right) u^{-\gamma_{12}} v^{-\gamma_{14}} V(1 ; 2,3)^{s-k} V(1 ; 3,4)^{k}
\end{aligned}
$$

where $V(i ; j, k)$ was defined in $(2.3)$ and

$$
\begin{aligned}
\hat{M}\left(\gamma_{12}, \gamma_{14} ; s, k\right)= & \Gamma\left(\gamma_{12}\right) \Gamma\left(\Delta_{1}-\gamma_{12}-\gamma_{14}\right) \Gamma\left(\gamma_{14}\right) \Gamma\left(\gamma_{12}+\frac{\Delta_{3}+\Delta_{4}-\Delta_{1}-\Delta_{2}}{2}\right) \\
& \Gamma\left(\frac{\Delta_{1}+\Delta_{2}-\Delta_{3}+\Delta_{4}}{2}-\gamma_{12}-\gamma_{14}\right) \Gamma\left(\gamma_{14}+\frac{\Delta_{2}+\Delta_{3}-\Delta_{1}-\Delta_{4}}{2}\right) \\
& M\left(\gamma_{12}, \gamma_{14} ; s, k\right), \\
\Delta_{1}= & 2 s+1, \quad \Delta_{2}=2, \quad \Delta_{3}=2, \quad \Delta_{4}=2 .
\end{aligned}
$$

We call $M\left(\gamma_{12}, \gamma_{14} ; s, k\right)$ a Mellin amplitude. The arguments of the $\Gamma$ functions are just the Mellin variables defined in (2.4).

In the limit $t \rightarrow \infty$ of the kinematics (3.1), the conformal cross-ratio $v$ acquires a monodromy $v \rightarrow v e^{2 \pi i}$. Furthermore

$$
u \approx 16 \sigma^{2}+O\left(\sigma^{3}\right), \quad v \approx 1-8 \sigma \cosh \rho+O\left(\sigma^{2}\right), \quad \sigma \rightarrow 0 .
$$


The polynomial growth of the Mellin amplitude is related to the Regge limit, in a manner that we explain next, following appendix $\mathrm{C}$ of [25]. Let us consider the limit

$$
\begin{aligned}
& \lim _{\sigma \rightarrow 0} \iint \frac{d \gamma_{12} d \gamma_{14}}{(2 \pi i)^{2}} M\left(\gamma_{12}, \gamma_{14} ; s, k\right) \Gamma\left(\gamma_{12}\right) \Gamma\left(\Delta_{1}-\gamma_{12}-\gamma_{14}\right) \\
& \Gamma\left(\gamma_{14}\right) e^{-2 \pi i \gamma_{14}} \Gamma\left(\gamma_{12}+\frac{\Delta_{3}+\Delta_{4}-\Delta_{1}-\Delta_{2}}{2}\right) \Gamma\left(\gamma_{14}+\frac{\Delta_{2}+\Delta_{3}-\Delta_{1}-\Delta_{4}}{2}\right) \\
& \Gamma\left(-\gamma_{12}-\gamma_{14}+\frac{\Delta_{1}+\Delta_{2}-\Delta_{3}+\Delta_{4}}{2}\right) \sigma^{-2 \gamma_{12}}(1-8 \sigma \cosh \rho)^{-\gamma_{14}} .
\end{aligned}
$$

The factor $e^{-2 \pi i \gamma_{14}}$ becomes very large in the regime $\gamma_{14} \rightarrow i \infty$. This is cancelled by the exponential decay of the $\Gamma$ functions. Let us suppose that the Mellin amplitude grows polynomially as $\gamma_{14}^{\alpha(s, k)} f\left(\gamma_{12}\right)$, when $\gamma_{14}$ is large and imaginary and $\gamma_{12}$ is fixed. In this regime we can rewrite (3.9) as

$$
\begin{aligned}
\sim & \int \frac{d \gamma_{12}}{2 \pi i} \Gamma\left(\gamma_{12}\right) \Gamma\left(\gamma_{12}+\frac{\Delta_{3}+\Delta_{4}-\Delta_{1}-\Delta_{2}}{2}\right) \sigma^{-2 \gamma_{12}} f\left(\gamma_{12}\right) \\
& \int_{M 1}^{\infty} \frac{d m_{1}}{2 \pi} m_{1}^{-2-2 \gamma_{12}+\Delta_{1}+\Delta_{2}+\alpha(s, k)} e^{i m_{1}\left(8 \sigma \cosh \rho+O\left(\sigma^{3}\right)\right)},
\end{aligned}
$$

where $M_{1}$ is an irrelevant large number. If we substitute $m_{1} \rightarrow \frac{m_{1}}{\sigma}$ we get that the integral (3.9) scales like $\sigma^{1-\Delta_{1}-\Delta_{2}-\alpha(s, k)}$. In order to compare (3.6) with (3.5), we should furthermore take into account the prefactor and the structures in (3.6), which scale with $\sigma$. Our conclusion is that $\alpha(s, k)=1-\lambda_{1}-k \leq 2-k$.

We can use the crossing symmetry equations

$$
\begin{aligned}
& \hat{M}\left(\gamma_{12}, \gamma_{14} ; s, k\right)=\sum_{k_{2}=k}^{s}(-1)^{k_{2}}\left(\begin{array}{c}
k_{2} \\
k
\end{array}\right) \hat{M}\left(2 s+1-k_{2}-\gamma_{12}-\gamma_{14}, \gamma_{14}-k+k_{2} ; s, k_{2}\right), \\
& \hat{M}\left(\gamma_{12}, \gamma_{14} ; s, k\right)=\hat{M}\left(\gamma_{14}, \gamma_{12} ; s, s-k\right) .
\end{aligned}
$$

to derive the following bounds on the polynomial growth of the Mellin amplitude

$$
\begin{aligned}
\lim _{\beta \rightarrow \infty} M\left(\gamma_{12}, \beta \gamma_{14} ; s, k\right) & \sim \beta^{\alpha_{1}(s, k)}, & & \alpha_{1}(s, k) \leq 2-k \\
\lim _{\beta \rightarrow \infty} M\left(\beta \gamma_{12}, \gamma_{14} ; s, k\right) & \sim \beta^{\alpha_{2}(s, k)}, & & \alpha_{2}(s, k) \leq 2-s+k \\
\lim _{\beta \rightarrow \infty} M\left(i \beta+\gamma_{12},-i \beta+\gamma_{14} ; s, k\right) & \sim \beta^{\alpha_{3}(s, k)}, & & \alpha_{3}(s, k) \leq 2+s .
\end{aligned}
$$

We can apply these bounds to the ansatz (2.7). We conclude that

$$
\begin{aligned}
& \lim _{\beta \rightarrow \infty} p\left(\gamma_{12}, \beta \gamma_{14} ; s, k\right) \sim \beta^{\eta_{1}(s, k)}, \quad \eta_{1}(s, k)=2+2 k+\alpha_{1}(s, k) \leq 4+k \\
& \lim _{\beta \rightarrow \infty} p\left(\beta \gamma_{12}, \gamma_{14} ; s, k\right) \sim \beta^{\eta_{2}(s, k)}, \quad \eta_{2}(s, k)=2+2 s-2 k+\alpha_{2}(s, k) \leq 4-k+s \\
& \lim _{\beta \rightarrow \infty} p\left(i \beta+\gamma_{12},-i \beta+\gamma_{14} ; s, k\right) \sim \beta^{\eta_{3}(s, k)}, \quad \eta_{3}(s, k) \leq 4+s .
\end{aligned}
$$

The solution that we found respects this bound. 




Figure 2. AdS contact diagram for $\left\langle j_{s} j_{\tilde{0}} j_{\tilde{0}} j_{\tilde{0}}\right\rangle$.

\subsection{The Regge limit of AdS contact diagrams for the parity even structure in $\left\langle j_{s} j_{\tilde{0}} j_{\tilde{0}} j_{\tilde{0}}\right\rangle$}

We will study the Regge limit of a generic AdS contact diagram for $\left\langle j_{s} j_{\tilde{0}} j_{\tilde{0}} j_{\tilde{0}}\right\rangle$ (see figure 2), using the methods of [26]. We use vectors $P_{i}$ and $Z_{i}$ in embedding space to describe the position and polarization vectors of an operator $O_{i}$ defined on the boundary of AdS. For tensor fields defined on the bulk of AdS, we use vectors $X_{i}$ and $W_{i}$ to denote the position and the polarization. The following identities are obeyed:

$$
Z_{i}^{2}=P_{i}^{2}=Z_{i} \cdot P_{i}=X_{i}^{2}+1=W_{i}^{2}=X_{i} \cdot W_{i}=0 .
$$

We denote the bulk to boundary propagator of a dimension $\Delta$ and spin $J$ field by $\Pi_{\Delta, J}(X, P ; W, Z)$. Its formula is

$$
\Pi_{\Delta, J}(X, P ; W, Z)=\mathcal{C}_{\Delta, J} \frac{((-2 P \cdot X)(W \cdot Z)+2(W \cdot P)(Z \cdot X))^{J}}{(-2 P \cdot X)^{\Delta+J}},
$$

where $\mathcal{C}_{\Delta, J}$ is a proportionality constant (whose value will not be relevant for us).

An important class of contact diagrams contributing to the parity even structure in $\left\langle j_{s} j_{\tilde{0}} j_{\tilde{0}} j_{\tilde{0}}\right\rangle$ is given by

$$
\begin{gathered}
\int_{\mathrm{AdS}} d X \Pi_{\Delta_{1}=s+1, s_{1}=s}\left(X, P_{1}, K, Z_{1}\right)(W \cdot \nabla)^{s_{2}} \Pi_{\Delta_{2}=2, s_{2}=0}\left(X, P_{2}\right) \\
(W \cdot \nabla)^{s_{3}} \Pi_{\Delta_{3}=2, s_{3}=0}\left(X, P_{3}\right) \Pi_{\Delta_{4}=2, s_{4}=0}\left(X, P_{4}\right),
\end{gathered}
$$

where $s_{1}=s_{2}+s_{3}$. There are other contact diagrams one can write by contracting more derivatives among the propagators, but such diagrams will diverge more in the Regge limit, which is the issue we wish to discuss here. The covariant derivative is given by

$$
\nabla_{A}=\frac{\partial}{\partial X^{A}}+X_{A}\left(X \cdot \frac{\partial}{\partial X}\right)+W_{A}\left(X \cdot \frac{\partial}{\partial W}\right) .
$$


The operator $K$ is given by

$$
\begin{aligned}
K_{A}= & \frac{d-1}{2}\left(\frac{\partial}{\partial W^{A}}+X_{A}\left(X \cdot \frac{\partial}{\partial W}\right)\right)+\left(W \cdot \frac{\partial}{\partial W}\right) \frac{\partial}{\partial W^{A}} \\
& +X_{A}\left(W \cdot \frac{\partial}{\partial W}\right)\left(X \cdot \frac{\partial}{\partial W}\right)-\frac{1}{2} W_{A}\left(\frac{\partial^{2}}{\partial W \cdot \partial W}+\left(X \cdot \frac{\partial}{\partial W}\right)\left(X \cdot \frac{\partial}{\partial W}\right)\right),
\end{aligned}
$$

where for our purposes $d=3$.

The following identity

$$
\begin{aligned}
& \Pi_{\Delta_{1}, s_{1}}\left(X, P_{1}, K, Z_{1}\right)(W \cdot \nabla)^{s_{2}} \Pi_{\Delta_{2}, s_{2}}\left(X, P_{2}\right)(W \cdot \nabla)^{s_{3}} \Pi_{\Delta_{3}, s_{3}}\left(X, P_{3}\right) \\
& =C\left(\Delta_{1}, \Delta_{2}, \Delta_{3}, s_{1}, s_{2}, s_{3}\right) D_{12}^{s_{2}} D_{13}^{s_{3}}\left(\Pi_{\Delta_{1}, 0}\left(X, P_{1}\right) \Pi_{\Delta_{2}+s_{2}, 0}\left(X, P_{2}\right) \Pi_{\Delta_{3}+s_{3}, 0}\left(X, P_{3}\right)\right) .
\end{aligned}
$$

is useful for us. $D_{i j}$ is an operator that only acts on the external points. It increases the spin at position $i$ by 1 and it decreases the conformal dimension at position $j$ by 1 . $C\left(\Delta_{1}, \Delta_{2}, \Delta_{3}, s_{1}, s_{2}, s_{3}\right)$ is a constant of proportionality, which will not be relevant for us. The precise definition of $D_{i j}$ is

$$
D_{i j}=\left(P_{j} \cdot Z_{i}\right) Z_{i} \cdot \frac{\partial}{\partial Z_{i}}-\left(P_{j} \cdot Z_{i}\right) P_{i} \cdot \frac{\partial}{\partial P_{i}}+\left(P_{j} \cdot P_{i}\right) Z_{i} \cdot \frac{\partial}{\partial P_{i}} .
$$

We confirmed the identity (3.24) for a few values of the external spins using Mathematica.

So, with the help of identity (3.24) we can perform the integration in (3.21) using only scalar propagators and afterwards we act with the differential operators $D_{12}$ and $D_{13}$. The AdS integral with only scalar propagators corresponds to a contact quartic scalar diagram, whose Mellin amplitude is a constant. Afterwards we act with the differential operators and obtain an expression in the form of (2.5).

Let us exemplify what we mean for the case of $\left\langle j_{2} j_{\tilde{0}} j_{\tilde{0}} j_{\tilde{0}}\right\rangle$. Let us take $s_{2}=1$ and $s_{3}=1 \mathrm{in}(3.21)$. Up to a proportionality constant, the contact diagram is given by

$$
\begin{aligned}
& D_{12} D_{13} \int_{\mathrm{AdS}} d X \Pi_{\Delta_{1}=3, s_{1}=0}\left(X, P_{1}\right) \Pi_{\Delta_{2}=3, s_{2}=0}\left(X, P_{2}\right) \Pi_{\Delta_{3}=3, s_{3}=0}\left(X, P_{3}\right) \Pi_{\Delta_{4}=2, s_{4}=0}\left(X, P_{4}\right) \\
& \sim D_{12} D_{13} \frac{x_{34}}{x_{23} x_{13}^{6} x_{24}^{5}} \iint \frac{d \gamma_{12} d \gamma_{14}}{(2 \pi i)^{2}} \Gamma\left(\gamma_{12}\right) \Gamma\left(3-\gamma_{12}-\gamma_{14}\right) \Gamma\left(\gamma_{14}\right) \\
& \quad \Gamma\left(\gamma_{12}-\frac{1}{2}\right) \Gamma\left(\frac{5}{2}-\gamma_{12}-\gamma_{14}\right) \Gamma\left(\gamma_{14}+\frac{1}{2}\right) u^{-\gamma_{12}} v^{-\gamma_{14}},
\end{aligned}
$$

where the $\sim$ symbol means that we neglected a numerical factor. We now act with the differential operators $D_{12}$ and $D_{13}$ and reorganise the result into the form (3.6), (3.7). ${ }^{5}$ For this contact diagram, we conclude that

$$
\begin{aligned}
& M\left(\gamma_{12}, \gamma_{14}, s=2, k=0\right)=\frac{\left(-4+\gamma_{12}\right)\left(3-8 \gamma_{14}+4 \gamma_{14}^{2}\right)}{\left(-4+\gamma_{12}+\gamma_{14}\right)} \\
& M\left(\gamma_{12}, \gamma_{14}, s=2, k=1\right)=\frac{-2\left(-2+\gamma_{12}\right)\left(-3+2 \gamma_{12}\right)\left(-3+2 \gamma_{14}\right)}{\left(-4+\gamma_{12}+\gamma_{14}\right)} \\
& M\left(\gamma_{12}, \gamma_{14}, s=2, k=2\right)=\frac{\gamma_{12}\left(3-8 \gamma_{12}+4 \gamma_{12}^{2}\right)}{\left(-4+\gamma_{12}+\gamma_{14}\right)}
\end{aligned}
$$

\footnotetext{
${ }^{5}$ The step where we gather different terms into the same contour may give rise to subtractions. These do not change our main conclusion, which is that any finite linear combination of AdS contact diagrams for $\left\langle j_{s} j_{\tilde{0}} j_{\tilde{0}} j_{\tilde{0}}\right\rangle$ with $s \geq 4$ does not obey the bound on chaos.
} 
This contact diagram obeys the chaos bounds (3.13), (3.14) and (3.15). We found that contact diagrams of the type (3.21) obey the bound on chaos for spin 2 , but violate the bound on chaos for spin $s \geq 4$.

Our goal is to investigate if there are extra solutions to crossing, conservation and Regge boundedness for $\left\langle j_{s} j_{\tilde{0}} j_{\tilde{0}} j_{\tilde{0}}\right\rangle$. AdS contact diagrams are solutions to the crossing equations, however they are not necessarily conserved, nor Regge bounded. To see that contact diagrams are not necessarily conserved, let us consider a generic contact diagram

$$
\int_{\text {AdS }} d X \Pi_{\Delta=s+1, s}\left(X, P_{1}, W, Z_{1}\right) J\left(X, P_{i}, K, Z_{i}\right)
$$

where we denoted by $J\left(X, P_{i}, W, Z_{i}\right)$ the dependence on the other AdS fields. It turns out that the action of the conservation operator $(2.10)$ on $\Pi_{\Delta=s+1, s}$ gives a pure gauge expression

$$
\begin{aligned}
& \frac{\partial}{\partial P} \cdot \mathcal{D}_{Z} \Pi_{\Delta=s+1, s}(X, P, W, Z) \\
& =-2^{-2-s} s^{2} W \cdot \nabla_{X}\left((-P \cdot X)^{-2 s-1}((-P \cdot X)(W \cdot Z)+(P \cdot W)(X \cdot Z))^{s-1}\right) \\
& \equiv W \cdot \nabla_{X} F(X, P, W, Z) .
\end{aligned}
$$

Thus,

$$
\begin{aligned}
& \frac{\partial}{\partial P_{1}} \cdot \mathcal{D}_{Z_{1}} \int_{\mathrm{AdS}} d X \Pi_{\Delta=s+1, s}\left(X, P_{1}, W, Z_{1}\right) J\left(X, P_{i}, W, Z_{i}\right) \\
& =-\int_{\mathrm{AdS}} d X F\left(X, P_{1}, W, Z_{1}\right) W \cdot \nabla_{X} J\left(X, P_{i}, K, Z_{i}\right)
\end{aligned}
$$

This vanishes only if $J\left(X, P_{i}, K, Z_{i}\right)$ is conserved in the bulk of AdS, i.e. a contact diagram involving a bulk to boundary propagator is conserved only when the bulk to boundary propagator is coupled to a conserved current. Clearly, this is not the case for a generic contact diagram (3.21).

So, we consider instead linear combinations of AdS contact diagrams. The most economical way of doing this is to notice that the Mellin transform of any contact diagram, or any linear combination of contact diagrams, can be written as

$$
\begin{aligned}
\hat{M}\left(\gamma_{12}, \gamma_{14} ; s, k\right)= & \Gamma\left(-k+\gamma_{14}\right) \Gamma\left(-k+\gamma_{14}+\frac{1}{2}\right) \Gamma\left(-s+\gamma_{13}\right) \\
& \times \Gamma\left(-s+\gamma_{13}+\frac{1}{2}\right) \Gamma\left(k-s+\gamma_{12}\right) \Gamma\left(k-s+\gamma_{12}+\frac{1}{2}\right) p_{d t}\left(\gamma_{12}, \gamma_{14} ; s, k\right) .
\end{aligned}
$$

where $p_{d t}\left(\gamma_{12}, \gamma_{14} ; s, k\right)$ is a polynomial. Let us explain this important formula. If we act with the differential operators on the scalar contact diagram, they will shift the arguments of the $\Gamma$ functions by integers. So, the Mellin transform of an AdS contact diagram will involve $6 \Gamma$ functions times a polynomial. The arguments of the $\Gamma$ functions are related to the operators that appear in the OPE of the external operators. Thus, we arrive at (3.33). Notice that $p_{d t}\left(\gamma_{12}, \gamma_{14} ; s, k\right)$ will eventually have zeros. 
The chaos bound for $p_{d t}\left(\gamma_{12}, \gamma_{14} ; s, k\right)$ is

$$
\begin{aligned}
\lim _{\beta \rightarrow \infty} p_{d t}\left(\gamma_{12}, \beta \gamma_{14} ; s, k\right) & \sim \beta^{\eta_{1}(s, k)}, \quad \eta_{1}(s, k)=2+2 k+\alpha_{1}(s, k) \leq 2+k \\
\lim _{\beta \rightarrow \infty} p_{d t}\left(\beta \gamma_{12}, \gamma_{14} ; s, k\right) & \sim \beta^{\eta_{2}(s, k)}, \quad \eta_{2}(s, k)=2+2 s-2 k+\alpha_{2}(s, k) \leq 2-k+s \\
\lim _{\beta \rightarrow \infty} p_{d t}\left(i \beta+\gamma_{12},-i \beta+\gamma_{14} ; s, k\right) & \sim \beta^{\eta_{3}(s, k)}, \quad \eta_{3}(s, k) \leq 2+s .
\end{aligned}
$$

We imposed crossing and conservation on (3.33). We find solutions that always violate the chaos bound, for all spins $s \geq 4$. For $s=2$ we find a solution that respects crossing, conservation and Regge boundedness, which is given by

$$
\begin{aligned}
p_{d t}\left(\gamma_{12}, \gamma_{14} ; s=2, k=0\right)= & \frac{\gamma_{12}^{4} \gamma_{14}}{9}+\frac{\gamma_{12}^{4}}{24}+\frac{\gamma_{12}^{3} \gamma_{14}^{2}}{9}-\frac{5 \gamma_{12}^{3} \gamma_{14}}{8}-\frac{5 \gamma_{12}^{3}}{12}-\frac{7 \gamma_{12}^{2} \gamma_{14}^{2}}{24} \\
& +\frac{35 \gamma_{12}^{2} \gamma_{14}}{36}+\frac{35 \gamma_{12}^{2}}{24}+\frac{7 \gamma_{12} \gamma_{14}^{2}}{72}-\frac{5 \gamma_{12} \gamma_{14}}{24} \\
& -\frac{25 \gamma_{12}}{12}+\frac{\gamma_{14}^{2}}{12}-\frac{\gamma_{14}}{4}+1, \\
p_{d t}\left(\gamma_{12}, \gamma_{14} ; s=2, k=1\right)= & -\frac{2 \gamma_{12}^{3} \gamma_{14}^{2}}{9}+\frac{5 \gamma_{12}^{3} \gamma_{14}}{4}-\frac{37 \gamma_{12}^{3}}{36}-\frac{2 \gamma_{12}^{2} \gamma_{14}^{3}}{9}+\frac{13 \gamma_{12}^{2} \gamma_{14}^{2}}{4} \\
& -\frac{331 \gamma_{12}^{2} \gamma_{14}}{36}+\frac{37 \gamma_{12}^{2}}{6}+\frac{5 \gamma_{12} \gamma_{14}^{3}}{4}-\frac{331 \gamma_{12} \gamma_{14}^{2}}{36}+\frac{77 \gamma_{12} \gamma_{14}}{4} \\
& -\frac{407 \gamma_{12}}{36}-\frac{37 \gamma_{14}^{3}}{36}+\frac{37 \gamma_{14}^{2}}{6}-\frac{407 \gamma_{14}}{36}+\frac{37}{6}, \\
p\left(\gamma_{12}, \gamma_{14} ; s=2, k=2\right)= & \frac{\gamma_{12}^{2} \gamma_{14}^{3}}{9}-\frac{7 \gamma_{12}^{2} \gamma_{14}^{2}}{24}+\frac{7 \gamma_{12}^{2} \gamma_{14}}{72}+\frac{\gamma_{12}^{2}}{12}+\frac{\gamma_{12} \gamma_{14}^{4}}{9}-\frac{5 \gamma_{12} \gamma_{14}^{3}}{8} \\
& +\frac{35 \gamma_{12} \gamma_{14}^{2}}{36}-\frac{5 \gamma_{12} \gamma_{14}}{24}-\frac{\gamma_{12}}{4}+\frac{\gamma_{14}^{4}}{24} \\
& -\frac{5 \gamma_{14}^{3}}{12}+\frac{35 \gamma_{14}^{2}}{24}-\frac{25 \gamma_{14}}{12}+1 .
\end{aligned}
$$

More explicitly, the spin 2 parity even contact term in position space is given by

$$
\left\langle j_{2} j_{\tilde{0}} j_{\tilde{0}} j_{\tilde{0}}\right\rangle=\left|x_{1}-x_{3}\right|^{-10}\left|x_{2}-x_{3}\right|^{3}\left|x_{2}-x_{4}\right|^{-7}\left|x_{3}-x_{4}\right|^{3} \sum_{k=0}^{s} f_{k}(u, v) w(1 ; 2,3)^{s-k} w(1 ; 3,4)^{k}
$$

where $w(i ; j, k)=\frac{\left(x_{i j}\right)_{\mu} x_{i k}^{2}}{x_{j k}^{2}}-\frac{\left(x_{i k}\right)_{\mu} x_{i j}^{2}}{x_{j k}^{2}}$ and the indices are symmetrized and their traces removed. For example $w(1 ; 2,3)^{2}=w(1 ; 2,3)_{\mu} w(1 ; 2,3)_{\nu}-\frac{\eta_{\mu \nu}}{3} w(1 ; 2,3)^{\rho} w(1 ; 2,3)_{\rho}$. Also, ${ }^{6}$

$$
\begin{aligned}
f_{k}(u, v)= & \int_{\frac{7}{3}-k-i \infty}^{\frac{7}{3}-k+i \infty} \frac{d \gamma_{12}}{2 \pi i} \int_{\frac{1}{3}+k-i \infty}^{\frac{1}{3}+k+i \infty} \frac{d \gamma_{14}}{2 \pi i} \hat{M}\left(\gamma_{12}, \gamma_{14}, s=2, k\right) u^{-\gamma_{12}} v^{-\gamma_{14}} \\
\hat{M}\left(\gamma_{12}, \gamma_{14} ; s=2, k\right)= & \Gamma\left(-k+\gamma_{14}\right) \Gamma\left(-k+\gamma_{14}+\frac{1}{2}\right) \Gamma\left(3-\gamma_{12}-\gamma_{14}\right) \\
& \times \Gamma\left(\frac{7}{2}-\gamma_{12}-\gamma_{14}\right) \Gamma\left(k-2+\gamma_{12}\right) \Gamma\left(k+\gamma_{12}-\frac{3}{2}\right) p_{d t}\left(\gamma_{12}, \gamma_{14} ; s=2, k\right),
\end{aligned}
$$

where $p_{d t}\left(\gamma_{12}, \gamma_{14} ; s=2, k\right)$ is given by $(3.37)$.

\footnotetext{
${ }^{6}$ There is some arbitrariness in the choice of the contour. What is important is that it passes to the right of the poles in $\gamma_{12}, \gamma_{13}$ and $\gamma_{14}$, see [21].
} 


\subsection{The Regge limit of AdS contact diagrams for the parity odd structure in} $\left\langle j_{s} j_{\tilde{0}} j_{\tilde{0}} j_{\tilde{0}}\right\rangle$

The parity odd structure is

$$
\begin{aligned}
\left\langle j_{s}\left(x_{1}\right) j_{\tilde{0}}\left(x_{2}\right) j_{\tilde{0}}\left(x_{3}\right) j_{\tilde{0}}\left(x_{4}\right)\right\rangle_{\text {odd }}= & \left|x_{1}-x_{3}\right|^{-4 s-2}\left|x_{2}-x_{3}\right|^{2 s-2}\left|x_{2}-x_{4}\right|^{-2 s-4}\left|x_{3}-x_{4}\right|^{2 s-2} \\
& \times \sum_{k=0}^{s-1} \iint \frac{d \gamma_{12} d \gamma_{14}}{(2 \pi i)^{2}} \hat{M}_{\text {odd }}\left(\gamma_{12}, \gamma_{14} ; s, k\right) u^{-\gamma_{12}} v^{-\gamma_{14}} \epsilon \\
& \times\left(Z_{1}, P_{1}, P_{2}, P_{3}, P_{4}\right) V(1 ; 2,3)^{s-1-k} V(1 ; 3,4)^{k} .
\end{aligned}
$$

We define the Mellin amplitude $M_{\text {odd }}\left(\gamma_{12}, \gamma_{14} ; s, k\right)$ in the following manner

$$
\begin{aligned}
\hat{M}_{\text {odd }}\left(\gamma_{12}, \gamma_{14} ; s, k\right)= & \Gamma\left(\gamma_{12}\right) \Gamma\left(\Delta_{1}-\gamma_{12}-\gamma_{14}\right) \Gamma\left(\gamma_{14}\right) \Gamma\left(\gamma_{12}+\frac{\Delta_{3}+\Delta_{4}-\Delta_{1}-\Delta_{2}}{2}\right) \\
& \Gamma\left(\frac{\Delta_{1}+\Delta_{2}-\Delta_{3}+\Delta_{4}}{2}-\gamma_{12}-\gamma_{14}\right) \\
& \Gamma\left(\gamma_{14}+\frac{\Delta_{2}+\Delta_{3}-\Delta_{1}-\Delta_{4}}{2}\right) M_{\text {odd }}\left(\gamma_{12}, \gamma_{14} ; s, k\right), \\
\Delta_{1}= & 2 s+1, \quad \Delta_{2}=3, \quad \Delta_{3}=3, \quad \Delta_{4}=3 .
\end{aligned}
$$

The following equations encapsulate crossing symmetry:

$$
\begin{aligned}
& \hat{M}_{\text {odd }}\left(\gamma_{12}, \gamma_{14} ; s, k\right)=\sum_{k_{2}=k}^{s-1}(-1)^{k_{2}}\left(\begin{array}{c}
k_{2} \\
k
\end{array}\right) \hat{M}_{\text {odd }}\left(2 s+1-k_{2}-\gamma_{12}-\gamma_{14}, \gamma_{14}-k+k_{2} ; s, k_{2}\right), \\
& \hat{M}_{\text {odd }}\left(\gamma_{12}, \gamma_{14} ; s, k\right)=\hat{M}_{\text {odd }}\left(\gamma_{14}, \gamma_{12} ; s, s-1-k\right) .
\end{aligned}
$$

Let us use the bound on chaos to derive a bound on the polynomial growth of the Mellin amplitude. Let us define the exponent $\alpha(s ; k)$ such that $\lim _{\beta \rightarrow \infty} M\left(\gamma_{12}, \beta \gamma_{14} ; s, k\right) \sim$ $\beta^{\alpha(s ; k)}$. In the Regge limit, the Mellin integral goes as $\sigma^{-2 s-3-\alpha(s ; k)}$. The prefactor times the structure goes as $\sigma^{3+2 s-k}$. So, (3.41) behaves as $\sigma^{-k-\alpha(s ; k)}$. By comparing with the bound on chaos (3.5) and using (3.43), (3.44) we conclude that

$$
\begin{aligned}
\lim _{\beta \rightarrow \infty} M_{\text {odd }}\left(\gamma_{12}, \beta \gamma_{14} ; s, k\right) & \sim \beta^{\alpha_{1}(s, k)}, & & \alpha_{1}(s, k) \leq 1-k \\
\lim _{\beta \rightarrow \infty} M_{\text {odd }}\left(\beta \gamma_{12}, \gamma_{14} ; s, k\right) & \sim \beta^{\alpha_{2}(s, k)}, & & \alpha_{2}(s, k) \leq 2-s+k \\
\lim _{\beta \rightarrow \infty} M_{\text {odd }}\left(i \beta+\gamma_{12},-i \beta+\gamma_{14} ; s, k\right) & \sim \beta^{\alpha_{3}(s, k)}, & & \alpha_{3}(s, k) \leq s .
\end{aligned}
$$

The Mellin amplitude of an AdS contact diagram of the type (3.41), or of a linear combination of contact diagrams, is given by

$$
\begin{aligned}
\hat{M}_{\text {odd }}\left(\gamma_{12}, \gamma_{14} ; s, k\right)= & \Gamma\left(\gamma_{12}+1+k-s\right) \Gamma\left(\gamma_{12}+\frac{1}{2}+k-s\right) \\
& \Gamma\left(\gamma_{14}-k\right) \Gamma\left(\gamma_{14}-k-\frac{1}{2}\right) \Gamma\left(\gamma_{13}+1-s\right) \\
& \Gamma\left(\gamma_{13}+\frac{1}{2}-s\right) p_{d t}\left(\gamma_{12}, \gamma_{14} ; s, k\right),
\end{aligned}
$$


where $\gamma_{13}=2 s+1-\gamma_{12}-\gamma_{14}$. The bound on chaos for $p_{d t}\left(\gamma_{12}, \gamma_{14} ; s, k\right)$ is

$$
\begin{aligned}
\lim _{\beta \rightarrow \infty} p_{d t}\left(\gamma_{12}, \beta \gamma_{14} ; s, k\right) & \sim \beta^{\lambda_{1}(s, k)}, & & \lambda_{1}(s, k) \leq 2+k \\
\lim _{\beta \rightarrow \infty} p_{d t}\left(\beta \gamma_{12}, \gamma_{14} ; s, k\right) & \sim \beta^{\lambda_{2}(s, k)}, & & \lambda_{2}(s, k) \leq 1+s-k \\
\lim _{\beta \rightarrow \infty} p_{d t}\left(i \beta+\gamma_{12},-i \beta+\gamma_{14} ; s, k\right) & \sim \beta^{\lambda_{3}(s, k)}, & & \lambda_{3}(s, k) \leq 1+s .
\end{aligned}
$$

$p_{d t}\left(\gamma_{12}, \gamma_{14} ; s, k\right)$ can be found by imposing crossing and conservation. We found that for $s \geq 4$ all solutions violate the bound on chaos.

However, for $s=2$ there is one solution that respects the bound on chaos. This solution is

$$
\begin{aligned}
& p_{d t}\left(\gamma_{12}, \gamma_{14} ; s=2, k=0\right)=\frac{\gamma_{12}^{2}}{4}+\frac{\gamma_{12} \gamma_{14}}{2}-\frac{5 \gamma_{12}}{4}-\frac{\gamma_{14}}{2}+1 \\
& p_{d t}\left(\gamma_{12}, \gamma_{14} ; s=2, k=1\right)=\frac{\gamma_{12} \gamma_{14}}{2}-\frac{\gamma_{12}}{2}+\frac{\gamma_{14}^{2}}{4}-\frac{5 \gamma_{14}}{4}+1
\end{aligned}
$$

\section{Open directions}

The methods developed in this paper potentially pave the way to compute all four point functions in conformal field theories with slightly broken higher spin symmetry. We believe that the next steps in this program are the following:

1. Compute $\left\langle j_{s} j_{0} j_{0} j_{0}\right\rangle$ in the quasi-boson theory. The conformal structures involved are the same as in this paper, so the calculation should be very similar.

2. Demonstrate that AdS contact diagrams are not present in $\left\langle j_{\tilde{0}} j_{\tilde{0}} j_{\tilde{0}} j_{\tilde{0}}\right\rangle$ and $\left\langle j_{2} j_{\tilde{0}} j_{\tilde{0}} j_{\tilde{0}}\right\rangle$ in the quasi-fermion theory using pure CFT arguments. The chaos bound allows for contact diagrams in $\left\langle j_{\tilde{0}} j_{\tilde{0}} j_{\tilde{0}} j_{\tilde{0}}\right\rangle$ and $\left\langle j_{2} j_{\tilde{0}} j_{\tilde{0}} j_{\tilde{0}}\right\rangle$. Their absence for $\left\langle j_{\tilde{0}} j_{\tilde{0}} j_{\tilde{0}} j_{\tilde{0}}\right\rangle$ was demonstrated in [10] using Feynman diagrams. It should be possible to give a pure CFT demonstration of this fact. The idea is to write down the higher spin Ward identity that connects $\left\langle j_{\tilde{0}} j_{\tilde{0}} j_{\tilde{0}} j_{\tilde{0}}\right\rangle$ and $\left\langle j_{2} j_{\tilde{0}} j_{\tilde{0}} j_{\tilde{0}}\right\rangle$, plug the AdS contact diagrams multiplied by arbitrary functions of the t'Hooft coupling and obtain that the only way for the Ward identity to be satisfied is if such functions vanish.

Let us mention some more ambitious problems:

1. Develop a code that computes all spinning four point functions in CFT's with slightly broken higher spin symmetry. Such a code should:

- generate the structures involved for a given four point function

- generate an ansatz for the Mellin transform, which should be a product of 6 Gamma functions (whose arguments are determined by the lightcone OPE, which is known) times polynomials

- impose crossing, pseudo-conservation and Regge boundedness to fix all the undetermined coefficients in the polynomials. 
What differs from what we did here is that for generic spins we should not use embedding space, since the conformal structures in embedding space are generically linearly dependent on each other. It is best to use conformal frame techniques instead. Concretely, one would need the 3 dimensional version of [19] (see also [18]).

2. Demonstrate that AdS contact diagrams are not present in four point functions in CFT's with slightly broken higher spin symmetry. As above, the hurdle should be in adapting our formalism to use the $3 \mathrm{~d}$ conformal frame.

Recently, a new formalism for correlators of conserved currents was proposed in [27]. The idea is to write the conformal structures in a helicity basis. It would be very interesting to apply this idea to correlators in CFT's with slightly broken higher spin symmetry.

Ultimately, one would like to understand higher spin symmetry from the point of view of the bulk of AdS. We hope that our CFT computations can be of some utility for this ultimate goal.

\section{Acknowledgments}

I am very grateful to Joao Penedones and Alexander Zhiboedov for suggesting me this problem and for all the help they provided me. All the errors are of course mine. Furthermore, I would like to thank discussions with Aditya Hebbar, Evgeny Skvortsov, Subham Chowdhury and Vasco Gonçalves. This work was partially supported by a grant from the Simons Foundation (Simons Collaboration on the Nonperturbative Bootstrap: 488649) and by the Swiss National Science Foundation through the project 200021-169132. This project has received funding from the European Research Council (ERC) under the European Union's Horizon 2020 research and innovation programme (grant agreement number 949077).

\section{A Bulk point limit}

Correlation functions of conformal field theories in Lorentzian signature may diverge even when none of the distances between the points vanish. At the moment a full classification of the singularity structure of correlation functions in conformal field theories does not exist.

One such singularity is the so called "bulk point singularity". In terms of cross ratios, we can obtain such a singularity in the following manner. In Lorentzian signature $z$ and $\bar{z}$ are independent real numbers. The four point function has branch points. When $z$ and $\bar{z}$ go around the branch points the four point function may develop a divergence when $z=\bar{z}$. More specifically, suppose $z$ goes around the branch point at $1, \bar{z}$ goes around $\infty$ and now take $z \rightarrow \bar{z}$. We generically expect the four point function to diverge in this limit. A detailed examination of the bulk point limit for a four point function of equal scalars was carried out in [20].

In the bulk point limit a $d$ dimensional conformal block where the external operators are scalars diverges as $\frac{1}{(z-\bar{z})^{d-3}}[20]$. For this reason it is expected that a generic 
nonperturbative four point function of scalars diverges as

$$
\langle\mathcal{O O O O}\rangle \sim \frac{1}{(z-\bar{z})^{d-3}} .
$$

However, when the CFT has a local bulk dual, then we expect the divergence to be more severe. For example, a contact quartic diagram in AdS diverges as

$$
\langle\mathcal{O O O O}\rangle \sim \frac{1}{(z-\bar{z})^{4 \Delta-3}}
$$

The plan for this section is the following. In A.1 we calculate the bulk point singularity of an AdS contact diagram for a scalar four point function of unequal primaries. The result is a trivial generalisation of (A.2), however to our knowledge its derivation had not appeared before in the literature. We need such a result in order to calculate the bulk point singularity of an AdS contact diagram for $\left\langle j_{s} j_{\tilde{0}} j_{\tilde{0}} j_{\tilde{0}}\right\rangle$, which we do in appendix A.2. Finally, in appendix A.3 we calculate the expected bulk point divergence of $\left\langle j_{s} j_{\tilde{0}} j_{\tilde{0}} j_{\tilde{0}}\right\rangle$ in CFT's with slightly broken higher spin symmetry. We assume that $\left\langle j_{s} j_{\tilde{0}} j_{\tilde{0}} j_{\tilde{0}}\right\rangle$ does not diverge more than conformal blocks in the bulk point limit. We conclude that AdS contact diagrams diverge more severely in the bulk point limit than what is expected for $\left\langle j_{s} j_{\tilde{0}} j_{\tilde{0}} j_{\tilde{0}}\right\rangle$ for $s \geq 2$ in CFT's with slightly broken higher spin symmetry. Thus, bulk point softness implies that we cannot add AdS contact diagrams to the solution to the pseudo-conservation equations that we found in section 2 .

Let us add a caveat. Our result for $\left\langle j_{s} j_{\tilde{0}} j_{\tilde{0}} j_{\tilde{0}}\right\rangle$ does not rely on assuming bulk point softness and is independent of it. Nevertheless, we choose to keep this appendix, because it was useful for us to think in terms of the bulk point limit in the early stages of our work, and maybe this can be of use to someone else.

\section{A.1 Bulk point singularity of an AdS contact diagram for a scalar four point function of unequal primaries}

A quartic contact diagram has a Mellin amplitude equal to 1 . We will use this to compute the bulk point divergence, proceeding similarly to section 7.5.1 in [21]. Upon analytic continuation, the diagram is given by

$$
\begin{aligned}
\frac{\left\langle\mathcal{O}_{1} \mathcal{O}_{2} \mathcal{O}_{3} \mathcal{O}_{4}\right\rangle}{p}= & \iint \frac{d \gamma_{12} d \gamma_{14}}{(2 \pi i)^{2}} \Gamma\left(\gamma_{12}\right) \Gamma\left(\gamma_{13}\right) \Gamma\left(\gamma_{14}\right) \Gamma\left(\gamma_{12}+a_{34}\right) \\
& \times \Gamma\left(\gamma_{13}+a_{24}\right) \Gamma\left(\gamma_{14}+a_{23}\right) u^{-\gamma_{12}} v^{-\gamma_{14}} \\
\rightarrow & \iint \frac{d \gamma_{12} d \gamma_{14}}{(2 \pi i)^{2}} \Gamma\left(\gamma_{12}\right) \Gamma\left(\gamma_{13}\right) \Gamma\left(\gamma_{14}\right) \Gamma\left(\gamma_{12}+a_{34}\right) \\
& \times \Gamma\left(\gamma_{13}+a_{24}\right) \Gamma\left(\gamma_{14}+a_{23}\right) u^{-\gamma_{12}} v^{-\gamma_{14}} e^{-2 \pi i\left(\gamma_{12}+\gamma_{14}\right)} \\
p= & \left|x_{1}-x_{3}\right|^{-2 \Delta_{1}}\left|x_{2}-x_{3}\right|^{-2 a_{23}}\left|x_{2}-x_{4}\right|^{-2 a_{24}-2 \Delta_{1}}\left|x_{3}-x_{4}\right|^{-2 a_{34}}
\end{aligned}
$$

where $a_{i j}=2\left(\Delta_{i}+\Delta_{j}\right)-\sum_{k} \Delta_{k}$ and $\gamma_{13}=\Delta_{1}-\gamma_{12}-\gamma_{14}$. The integral diverges when $\gamma_{12}$ and $\gamma_{14}$ have a very big and positive imaginary part. We can use Stirling's approximation 
for the $\Gamma$ functions. Indeed suppose we take $\gamma_{12}=i s \beta$ and $\gamma_{14}=i s(1-\beta)$. Then for very large $s$ we have

$$
\begin{aligned}
\left\langle\mathcal{O}_{1} \mathcal{O}_{2} \mathcal{O}_{3} \mathcal{O}_{4}\right\rangle \approx & p \int_{s_{0}}^{\infty} \frac{d s}{s} \int_{0}^{1} d \beta s^{\frac{\sum_{i} \Delta_{i}}{2}-1} f(\beta) \\
& \times \exp (i s(-2(\beta-1) \log (1-\beta)+2 \beta \log (\beta)-\beta \log (u)+(\beta-1) \log (v))),
\end{aligned}
$$

where $f(\beta)$ is a function of $\beta$ that will not play any role. The integral has a saddle point for $\beta \rightarrow \beta_{s}=\frac{\sqrt{u}}{\sqrt{u}+\sqrt{v}}$. In that case the exponential dependence of the integrand becomes $e^{i s\left(\frac{(\sqrt{u}+\sqrt{v})^{2}}{\sqrt{u} \sqrt{v}}\left(\beta-\beta_{s}\right)^{2}-2 \log (\sqrt{u}+\sqrt{v})\right)}$. The integral in $\beta$ is Gaussian and can be readily evaluated. Furthermore, the phase is stationary when $\sqrt{u}+\sqrt{v}=1$. In that case we have $\log (\sqrt{u}+\sqrt{v}) \sim(z-\bar{z})^{2}$. So, we conclude that

$$
\left\langle\mathcal{O}_{1} \mathcal{O}_{2} \mathcal{O}_{3} \mathcal{O}_{4}\right\rangle \sim \int_{s_{0}}^{\infty} \frac{d s}{s} s^{\frac{\sum_{i} \Delta_{i}}{2}-\frac{3}{2}} e^{i s(z-\bar{z})^{2}} \sim \frac{1}{(z-\bar{z})^{\sum_{i} \Delta_{i}-3}}
$$

\section{A.2 Bulk point singularity of AdS contact diagrams for $\left\langle j_{s} j_{\tilde{0}} j_{\tilde{0}} j_{\tilde{0}}\right\rangle$}

Identity (3.24) allows us to obtain spinning contact AdS diagrams from scalar contact AdS diagrams. So, with the help of identity (3.24) we can perform the integration in (3.21) using only scalar propagators and afterwards we act with the differential operators $D_{12}$ and $D_{13}$. The scalar propagators cause a divergence like $\frac{1}{(z-\bar{z}) \sum_{i} \Delta_{i}-3+s}$, see formula (A.5). After acting with the differential operators, we find that the bulk point divergence of the integral (3.21) is $\frac{1}{(z-\bar{z})^{\sum_{i} \Delta_{i}-3+3 s}}=\frac{1}{(z-\bar{z})^{4 s+4}}$.

\section{A.3 Bulk point singularity of $\left\langle j_{s} j_{\tilde{0}} j_{\tilde{0}} j_{\tilde{0}}\right\rangle$ in CFT's with slightly broken higher spin symmetry}

Conformal field theories with slightly broken higher spin symmetry have an infinite number of light single trace operators. For this reason, they are not expected to be dual to a local theory in AdS. Thus, their bulk point singularity should not be enhanced with respect to that of an individual conformal block.

We want to calculate the bulk point divergence of $\left\langle j_{s} j_{\tilde{0}} j_{\tilde{0}} j_{\tilde{0}}\right\rangle$. For our discussion, it is useful to introduce the operator

$$
d_{11}=\left(P_{1} \cdot P_{2}\right) Z_{1} \cdot \frac{\partial}{\partial P_{2}}-\left(Z_{1} \cdot P_{2}\right) P_{1} \cdot \frac{\partial}{\partial P_{2}}-\left(Z_{1} \cdot Z_{2}\right) P_{1} \cdot \frac{\partial}{\partial Z_{2}}+\left(P_{1} \cdot Z_{2}\right) Z_{1} \cdot \frac{\partial}{\partial Z_{2}},
$$

where we used embedding space coordinates [14]. This operator acts on conformal blocks where the operator exchanged is symmetric and traceless. It increases the spin of the operator in position 1 by 1 and it decreases its conformal dimension by 1 also. It turns out that $d_{11}^{s}(z-\bar{z})^{a} \sim(z-\bar{z})^{a-2 s}$, i.e. the action of $d_{11}^{s}$ increases the divergence by a power of $2 s$. For this reason, we expect the divergence of $\left\langle j_{s} j_{\tilde{0}} j_{\tilde{0}} j_{\tilde{0}}\right\rangle$ to be

$$
\left\langle j_{s} j_{\tilde{0}} j_{\tilde{0}} j_{\tilde{0}}\right\rangle \sim \frac{1}{(z-\bar{z})^{2 s}}
$$


since the scalar conformal block diverges logarithmically. We could have picked other differential operators than $d_{11}$ to create spin from the scalar conformal block. Since such operators only contain first derivatives of $P_{i}$ (and not higher derivatives), they lead to the same divergence (A.7).

\section{B Algorithm for computing $\left\langle j_{s} j_{\tilde{0}} j_{\tilde{0}} j_{\tilde{0}}\right\rangle$ in position space}

We will implement an algorithm in position space to calculate $\left\langle j_{s} j_{\tilde{0}} j_{\tilde{0}} j_{\tilde{0}}\right\rangle_{c b}$. The results match with the Mellin space calculation.

$\left\langle j_{s} j_{\tilde{0}} j_{\tilde{0}} j_{\tilde{0}}\right\rangle_{c b}$ is constrained by conformal symmetry, crossing, consistency with OPE and the pseudo-conservation equation that $j_{s}$ obeys. Conformal symmetry implies that

$$
\left\langle j_{s} j_{\tilde{0}} j_{\tilde{0}} j_{\tilde{0}}\right\rangle_{c b}=p \sum_{j=0}^{s} f_{j}(u, v) w(1 ; 2,3)^{j} w(1 ; 3,4)^{s-j}
$$

where

$$
\begin{array}{r}
p \equiv \frac{\left(x_{23}^{2} x_{24}^{2} x_{34}^{2}\right)^{\frac{s}{3}-\frac{5}{6}}}{\left(x_{12}^{2} x_{13}^{2} x_{14}^{2}\right)^{\frac{2 s}{3}+\frac{1}{3}}}, \quad u \equiv \frac{x_{12}^{2} x_{34}^{2}}{x_{13}^{2} x_{24}^{2}}, \quad v \equiv \frac{x_{14}^{2} x_{23}^{2}}{x_{13}^{2} x_{24}^{2}}, \\
w(i ; j, k) \equiv\left(x_{i j}\right)_{\mu} \frac{x_{i k}^{2}}{x_{j k}^{2}}-\left(x_{i k}\right)_{\mu} \frac{x_{i j}^{2}}{x_{j k}^{2}}
\end{array}
$$

and we use the notation $\left(x_{i j}\right)_{\mu}=\left(x_{i}\right)_{\mu}-\left(x_{j}\right)_{\mu}, x_{i j}=\left|x_{i}-x_{j}\right|$. The indices are symmetric and traceless. $f_{j}(u, v)$ is a function of the cross ratios not determined by conformal symmetry.

We write the following ansatz.

$$
f_{j}(u, v)=\frac{u^{a(j)} v^{b(j)}}{(1+\sqrt{u}+\sqrt{v})^{s}} \sum_{n_{j}=0}^{N(j)} \sum_{m_{j}=0}^{M(j)} c_{n_{j}, m_{j}} u^{\frac{n_{j}}{2}} v^{\frac{m_{j}}{2}}
$$

where $c_{n_{j}, m_{j}}$ are parameters that will be fixed by crossing and the pseudo-conservation equation. The values of $a(j), b(j), M(j)$ and $N(j)$ will follow from consistency with the operator product expansion.

Let us motivate the preceding ansatz. The spinning four point functions are related to the scalar four point functions by slightly broken higher spin Ward identities. The scalar four point function is a linear combination of powers of $u$ and of $v$. So, it is natural that $f_{j}(u, v)$ is made up of powers of $u$ and of $v$.

We will see below that the contribution to the operator product expansion of a certain operator goes as $\sim u^{\frac{\tau}{2}}$, where $\tau$ is the twist, which is defined as the conformal dimension minus the spin. Since all operator dimensions are integers, it is natural that the ansatz involves semi-integer powers of $u$ and of $v$. The denominator $\frac{1}{(1+\sqrt{u}+\sqrt{v})^{s}}$ diverges in the bulk point limit as $\frac{1}{(z-\bar{z})^{2 s}}$, which agrees with the discussion in A.3.

We can fix $a(j), b(j), N(j), M(j)$ by consistency with the lightcone operator product expansion. Let us explain the general idea. Consider two primary operators $O_{\mu_{1} \ldots \mu_{1}}(x)$, 
$O_{\nu_{1} \ldots \nu_{l_{2}}}(0)$ of conformal dimensions $\Delta_{1}$ and $\Delta_{2}$ and spins $l_{1}$ and $l_{2}$ and suppose they exchange a primary operator $O_{\rho_{1} \ldots \rho_{l}}$ of dimension $\Delta$ and spin $l$. The most singular term due to $O_{\rho_{1} \ldots \rho_{l}}$ that can appear in the lightcone operator product expansion is

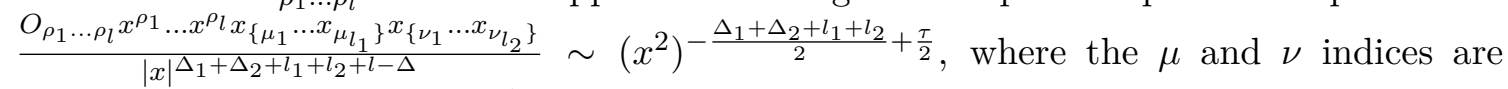
traceless symmetric and $\tau=\Delta-l$.

For $\left\langle j_{s} j_{\tilde{0}} j_{\tilde{0}} j_{\tilde{0}}\right\rangle$ the primary operators exchanged can have twist 1 (higher spin currents), $3+2 n$ (double traces $\left[j_{s}, j_{\tilde{0}}\right]$ ) and $4+2 n$ (double traces $\left[j_{\tilde{0}}, j_{\tilde{0}}\right]$ ), where $n$ is a nonnegative integer. There is no primary operator of twist 2 being exchanged. This is an important condition that we impose in our algorithm.

More explicitly

$$
\begin{aligned}
& j_{s}(x) j_{\tilde{0}}(0) \sim\left(x^{2}\right)^{-s-1} j_{s^{\prime}}+\left(x^{2}\right)^{-s}\left[j_{s}, j_{\tilde{0}}\right]+\left(x^{2}\right)^{-s+\frac{1}{2}}\left[j_{\tilde{0}}, j_{\tilde{0}}\right], \\
& j_{\tilde{0}}(x) j_{\tilde{0}}(0) \sim\left(x^{2}\right)^{-\frac{3}{2}} j_{s^{\prime}}+\left(x^{2}\right)^{-\frac{1}{2}}\left[j_{s}, j_{\tilde{0}}\right]+\left(x^{2}\right)^{0}\left[j_{\tilde{0}}, j_{\tilde{0}}\right],
\end{aligned}
$$

where we wrote the most singular powers of the distance that can appear in the lightcone operator product expansion. Our ansatz (B.3) needs to be compatible with (B.4), (B.5). This fixes $a(j), b(j), N(j), M(j)$.

The final ingredient is compatibility with pseudo-conservation. $\partial \cdot j_{s}$ can have contributions coming from $\left[j_{s_{1}}, j_{\tilde{0}}\right]$ and $\left[j_{s_{1}}, j_{s_{2}}\right]$. Only the former matter since we are interested in $\left\langle j_{s} j_{\tilde{0}} j_{\tilde{0}} j_{\tilde{0}}\right\rangle$. More precisely,

$$
\partial \cdot j_{s} \supset \sum_{s_{1}=2}^{s-2} \sum_{m=0}^{s-s_{1}-1} c_{m} \partial^{m} j_{s_{1}} \partial^{s-s_{1}-1-m} j_{\tilde{0}} .
$$

Since the right-hand side must be a conformal primary, this implies [8]

$$
c_{m}=\frac{-\left(m-s+s_{1}\right)\left(m-s+s_{1}-1\right)}{m\left(m+2 s_{1}\right)} c_{m-1} .
$$

Thus $\left\langle\partial \cdot j_{s} j_{\tilde{0}} j_{\tilde{0}} j_{\tilde{0}}\right\rangle$ is a linear combination of terms of type $\partial^{n_{1}}\left\langle j_{\tilde{0}} j_{\tilde{0}}\right\rangle \partial^{n_{2}}\left\langle j_{s_{1}} j_{\tilde{0}} j_{\tilde{0}}\right\rangle$.

Crossing and compatibility with pseudo-conservation fix all coefficients in (B.3) up to a number. This number is related to the normalizaton of $j_{s}$. In fact we did not even need to input formula (B.7), we kept the coefficients $c_{m}$ as unknowns and our algorithm correctly returns (B.7). This serves as a check on our results. We checked that the algorithm fixes the solution for $s=2, \ldots, 14$. Afterwards the computation becomes heavy for our laptop.

\section{Mixed Fourier transform}

We will solve the higher spin Ward identities to compute $\left\langle j_{2} j_{\tilde{0}} j_{\tilde{0}} j_{\tilde{0}}\right\rangle$. This is a rederivation of the main result of [11]. Our method involves the use of a mixed Fourier transform, see [27] and [28].

We use the metric $d s^{2}=-d x^{-} d x^{+}+d y^{2}$. We will take all indices lowered and in the minus component. We will study the action of the charge

$$
Q=\sqrt{\tilde{N}} \alpha_{4} \int_{x^{+}=\text {const. }} d x^{-} d y j_{----}
$$


on the four point function $\left\langle j_{\tilde{0}} j_{\tilde{0}} j_{\tilde{0}} j_{\tilde{0}}\right\rangle$. We make use of equations $[5,10]$

$$
\begin{aligned}
& \partial \cdot j_{4}=\alpha \frac{\tilde{\lambda}}{\sqrt{\tilde{N}} \sqrt{1+\tilde{\lambda}^{2}}}\left(: \partial_{-} j_{\tilde{0}} j_{2}:-\frac{2}{5}: j_{\tilde{0}} \partial_{-} j_{2}:\right), \\
& {\left[Q, j_{\tilde{0}}\right]=\partial_{-}^{3} j_{\tilde{0}}+\frac{\beta}{\sqrt{1+\tilde{\lambda}^{2}}}\left(\partial_{-} \partial_{-} j_{-y}-\partial_{-} \partial_{y} j_{--}\right) \text {. }}
\end{aligned}
$$

$\alpha, \alpha_{4}$ and $\beta$ are numerical coefficients that can be obtained from solving Ward identities at the level of three point functions. ${ }^{7}$ We will not need their precise value in what follows.

The scalar four point function obeys the slightly broken spin 4 Ward identity

$$
\left\langle\left[Q, j_{\tilde{0}}\right] j_{\tilde{0}} j_{\tilde{0}} j_{\tilde{0}}\right\rangle+\ldots=\sqrt{\tilde{N}} \alpha_{4} \int d^{3} x\left\langle\partial \cdot j_{4}(x) j_{\tilde{0}} j_{\tilde{0}} j_{\tilde{0}} j_{\tilde{0}}\right\rangle
$$

where by ... we mean the permutations (12), (13), (14). Note that

$$
\left\langle j_{\tilde{0}} j_{\tilde{0}} j_{\tilde{0}} j_{\tilde{0}}\right\rangle=\left\langle j_{\tilde{0}} j_{\tilde{0}} j_{\tilde{0}} j_{\tilde{0}}\right\rangle_{\text {disc }}+\frac{1}{N}\left\langle j_{\tilde{0}} j_{\tilde{0}} j_{\tilde{0}} j_{\tilde{0}}\right\rangle_{f f}
$$

where $\left\langle j_{\tilde{0}} j_{\tilde{0}} j_{\tilde{0}} j_{\tilde{0}}\right\rangle_{f f}$ denotes the connected piece in the free fermion theory and $\left\langle j_{\tilde{0}} j_{\tilde{0}} j_{\tilde{0}} j_{\tilde{0}}\right\rangle_{\text {disc }}$ denotes the disconnected piece. The disconnected piece obeys

$$
\left\langle\partial^{3} j_{\tilde{0}} j_{\tilde{0}} j_{\tilde{0}} j_{\tilde{0}}\right\rangle_{\text {disc }}+\ldots=0
$$

where we summed over all permutations. For this reason the disconnected piece drops out of (C.4). Using our ansatz (2.1) we conclude that

$$
\begin{aligned}
\left\langle\left[Q, j_{\tilde{0}}\right] j_{\tilde{0}} j_{\tilde{0}} j_{\tilde{0}}\right\rangle+\ldots= & \frac{1}{\tilde{N}}\left\langle\partial^{3} j_{\tilde{0}} j_{\tilde{0}} j_{\tilde{0}} j_{\tilde{0}}\right\rangle_{f f}+\frac{\beta}{\tilde{N}\left(1+\tilde{\lambda}^{2}\right)}\left(\left\langle\left(\partial_{-} \partial_{-} j_{-y}-\partial_{-} \partial_{y} j_{--}\right) j_{\tilde{0}} j_{\tilde{0}} j_{\tilde{0}}\right\rangle_{f f}\right. \\
& \left.+\tilde{\lambda}\left\langle\left(\partial_{-} \partial_{-} j_{-y}-\partial_{-} \partial_{y} j_{--}\right) j_{\tilde{0}} j_{\tilde{0}} j_{\tilde{0}}\right\rangle_{c b}\right)+\ldots
\end{aligned}
$$

From the Ward identities in the free fermion theory this becomes

$$
\begin{aligned}
\left\langle\left[Q, j_{\tilde{0}}\right] j_{\tilde{0}} j_{\tilde{0}} j_{\tilde{0}}\right\rangle+\ldots= & -\frac{\tilde{\lambda}^{2} \beta}{\tilde{N}\left(1+\tilde{\lambda}^{2}\right)}\left\langle\left(\partial_{-} \partial_{-} j_{-y}-\partial_{-} \partial_{y} j_{--}\right) j_{\tilde{0}} j_{\tilde{0}} j_{\tilde{0}}\right\rangle_{f f} \\
& +\frac{\tilde{\lambda} \beta}{\tilde{N}\left(1+\tilde{\lambda}^{2}\right)}\left\langle\left(\partial_{-} \partial_{-} j_{-y}-\partial_{-} \partial_{y} j_{--}\right) j_{\tilde{0}} j_{\tilde{0}} j_{\tilde{0}}\right\rangle_{c b}+\ldots
\end{aligned}
$$

Using (C.2) in the right-hand side of (C.4) we get

$$
\begin{aligned}
\sqrt{\tilde{N}} \alpha_{4} \int d^{3} x\left\langle\partial \cdot j_{4}(x) j_{\tilde{0}} j_{\tilde{0}} j_{\tilde{0}} j_{\tilde{0}}\right\rangle= & \alpha \alpha_{4} \frac{\tilde{\lambda}}{\sqrt{1+\tilde{\lambda}^{2}}} \int d^{3} x\left(\left\langle\partial_{-} j_{\tilde{0}}(x) j_{\tilde{0}}\right\rangle\left\langle j_{2}(x) j_{\tilde{0}} j_{\tilde{0}} j_{\tilde{0}}\right\rangle\right. \\
& \left.-\frac{2}{5}\left\langle j_{\tilde{0}}(x) j_{\tilde{0}}\right\rangle\left\langle\partial_{-} j_{2}(x) j_{\tilde{0}} j_{\tilde{0}} j_{\tilde{0}}\right\rangle+\ldots\right)
\end{aligned}
$$

We use the decomposition (2.1) to obtain that (C.9) is equal to

$$
\begin{aligned}
& \alpha \alpha_{4} \frac{\tilde{\lambda}}{\tilde{N}\left(1+\tilde{\lambda}^{2}\right)} \int d^{3} x\left(\left\langle\partial_{-} j_{\tilde{0}}(x) j_{\tilde{0}}\right\rangle\left\langle j_{2}(x) j_{\tilde{0}} j_{\tilde{0}} j_{\tilde{0}}\right\rangle_{f f}-\frac{2}{5}\left\langle j_{\tilde{0}}(x) j_{\tilde{0}}\right\rangle\left\langle\partial_{-} j_{2}(x) j_{\tilde{0}} j_{\tilde{0}} j_{\tilde{0}}\right\rangle_{f f}+\ldots\right) \\
& +\alpha \alpha_{4} \frac{\tilde{\lambda}^{2}}{\tilde{N}\left(1+\tilde{\lambda}^{2}\right)} \int d^{3} x\left(\left\langle\partial_{-} j_{\tilde{0}}(x) j_{\tilde{0}}\right\rangle\left\langle j_{2}(x) j_{\tilde{0}} j_{\tilde{0}} j_{\tilde{0}}\right\rangle_{c b}-\frac{2}{5}\left\langle j_{\tilde{0}}(x) j_{\tilde{0}}\right\rangle\left\langle\partial_{-} j_{2}(x) j_{\tilde{0}} j_{\tilde{0}} j_{\tilde{0}}\right\rangle_{c b}+\ldots\right)
\end{aligned}
$$

\footnotetext{
${ }^{7}$ We normalised the charge such that the coefficient multiplying $\partial_{-}^{3} j_{\tilde{0}}$ in (C.3) is 1 .
} 
Let us equate (C.8) and (C.10). We see that the dependence on $\tilde{N}$ and $\tilde{\lambda}$ matches on both sides provided

$$
\begin{aligned}
& \beta\left\langle\left(\partial_{-} \partial_{-} j_{-y}-\partial_{-} \partial_{y} j_{--}\right) j_{\tilde{0}} j_{\tilde{0}} j_{\tilde{0}}\right\rangle_{f f}+\ldots \\
& \quad=-\alpha \alpha_{4} \int d^{3} x\left(\left\langle\partial_{-} j_{\tilde{0}} j_{\tilde{0}}\right\rangle\left\langle j_{2} j_{\tilde{0}} j_{\tilde{0}} j_{\tilde{0}}\right\rangle_{c b}-\frac{2}{5}\left\langle j_{\tilde{0}} j_{\tilde{0}}\right\rangle\left\langle\partial j_{2} j_{\tilde{0}} j_{\tilde{0}} j_{\tilde{0}}\right\rangle_{c b}+\ldots\right), \\
& \beta\left\langle\left(\partial_{-} \partial_{-} j_{-y}-\partial_{-} \partial_{y} j_{--}\right) j_{\tilde{0}} j_{\tilde{0}} j_{\tilde{0}}\right\rangle_{c b}+\ldots \\
& =\alpha \alpha_{4} \int d^{3} x\left(\left\langle\partial_{-} j_{\tilde{0}} j_{\tilde{0}}\right\rangle\left\langle j_{2} j_{\tilde{0}} j_{\tilde{0}} j_{\tilde{0}}\right\rangle_{f f}-\frac{2}{5}\left\langle j_{\tilde{0}} j_{\tilde{0}}\right\rangle\left\langle\partial j_{2} j_{\tilde{0}} j_{\tilde{0}} j_{\tilde{0}}\right\rangle_{f f}+\ldots\right) .
\end{aligned}
$$

We solved (C.11) and (C.12) using a mixed Fourier transform. We define the mixed Fourier transform of a four point function $\left\langle\mathcal{O}_{1}\left(x_{1}\right) \mathcal{O}_{2}\left(x_{2}\right) \mathcal{O}_{3}\left(x_{3}\right) \mathcal{O}_{4}\left(x_{4}\right)\right\rangle$ as

$$
\left\langle\mathcal{O}_{1}\left(x_{1}\right) \mathcal{O}_{2}\left(x_{2}\right) \mathcal{O}_{3}\left(x_{3}\right) \mathcal{O}_{4}\left(x_{4}\right)\right\rangle \rightarrow \int \frac{d^{3} x_{2} d^{3} x_{3}}{(2 \pi i)^{2}}\left\langle\mathcal{O}_{1}(0) \mathcal{O}_{2}\left(x_{2}\right) \mathcal{O}_{3}\left(x_{3}\right) \mathcal{O}_{4}(\infty)\right\rangle e^{i\left(p_{2} \cdot x_{2}+p_{3} \cdot x_{3}\right)}
$$

The advantage of the mixed Fourier transform with respect to a usual Fourier transform is that by placing an operator at the origin and another one at $\infty$ we take advantage of conformal symmetry.

In mixed Fourier space we can get rid of the integrals in equations (C.11) and (C.12). For example, it is simple to see that the mixed Fourier transform of $\int d^{3} x\left\langle j_{\tilde{0}}(x) j_{\tilde{0}}\right\rangle\left\langle j_{2}(x) j_{\tilde{0}} j_{\tilde{0}} j_{\tilde{0}}\right\rangle$ is equal to

$$
\begin{aligned}
& \int d^{3} x\left\langle j_{\tilde{0}}(x) j_{\tilde{0}}\left(x_{1}\right)\right\rangle\left\langle j_{2}(x) j_{\tilde{0}}\left(x_{2}\right) j_{\tilde{0}}\left(x_{3}\right) j_{\tilde{0}}\left(x_{4}\right)\right\rangle \\
& \rightarrow\left(\int d^{3} x\left\langle j_{\tilde{0}}(x) j_{\tilde{0}}(0)\right\rangle e^{i\left(p_{2}+p_{3}\right) \cdot x}\right) \iint d^{3} x_{2} d^{3} x_{3} e^{i\left(p_{2} \cdot x_{2}+p_{3} \cdot x_{3}\right)}\left\langle j_{2}(0) j_{\tilde{0}}\left(x_{2}\right) j_{\tilde{0}}\left(x_{3}\right) j_{\tilde{0}}(\infty)\right\rangle
\end{aligned}
$$

which is just a product of mixed Fourier transforms.

It turns out that $\left\langle j_{2} j_{\tilde{0}} j_{\tilde{0}} j_{\tilde{0}}\right\rangle_{f f}$ is very simple in mixed Fourier space. Let us define $u_{p}=\frac{p_{2}^{2}}{p_{1}^{2}}, v_{p}=\frac{p_{3}^{2}}{p_{1}^{2}}$, where $p_{1}=-p_{2}-p_{3}$. Then,

$$
\begin{aligned}
\left\langle T_{\mu \nu}(0) j_{\tilde{0}}\left(p_{2}\right) j_{\tilde{0}}\left(p_{3}\right) j_{\tilde{0}}(\infty)\right\rangle_{f f}= & \frac{f\left(u_{p}, v_{p}\right)}{p_{1}^{4}}\left(\left(p_{2}\right)_{(\mu} \epsilon_{\nu) \alpha \beta}\left(p_{2}\right)^{\alpha}\left(p_{3}\right)^{\beta}\right) \\
& +\frac{f\left(v_{p}, u_{p}\right)}{p_{1}^{4}}\left(\left(p_{3}\right)_{(\mu} \epsilon_{\nu) \alpha \beta}\left(p_{3}\right)^{\alpha}\left(p_{2}\right)^{\beta}\right)
\end{aligned}
$$

where $f\left(u_{p}, v_{p}\right)=\frac{32}{3} \pi^{2}\left(-\frac{1}{u_{p}}+\frac{1}{v_{p}}-\frac{1}{u_{p} v_{p}}\right)$. Plugging this into (C.11) and (C.12) we obtain

$$
\begin{aligned}
\left\langle T_{\mu \nu}(0) j_{\tilde{0}}\left(p_{2}\right) j_{\tilde{0}}\left(p_{3}\right) j_{\tilde{0}}(\infty)\right\rangle_{c b}= & \frac{1}{\left|p_{1}\right|^{3}}\left(\left(p_{2}\right)_{(\mu}\left(p_{3}\right)_{\nu)}-\frac{p_{2} \cdot p_{3}}{3} \eta_{\mu \nu}\right) f_{1}\left(u_{p}, v_{p}\right) \\
& +\frac{1}{\left|p_{1}\right|^{3}}\left(\left(p_{2}\right)_{\mu}\left(p_{2}\right)_{\nu}-\frac{p_{2}^{2}}{3} \eta_{\mu \nu}\right) f_{2}\left(u_{p}, v_{p}\right) \\
& +\frac{1}{\left|p_{1}\right|^{3}}\left(\left(p_{3}\right)_{\mu}\left(p_{3}\right)_{\nu}-\frac{p_{3}^{2}}{3} \eta_{\mu \nu}\right) f_{2}\left(v_{p}, u_{p}\right)
\end{aligned}
$$


where

$$
\begin{aligned}
& f_{1}\left(u_{p}, v_{p}\right)=\frac{1}{2}\left(\frac{u_{p}}{v_{p}}+\frac{v_{p}}{u_{p}}\right)+\left(\frac{1}{u_{p}}+\frac{1}{v_{p}}\right)-\frac{3}{2 u_{p} v_{p}}, \\
& f_{2}\left(u_{p}, v_{p}\right)=\frac{u_{p}}{4 v_{p}}+\frac{v_{p}}{4 u_{p}}+\frac{1}{4 u_{p} v_{p}}+\frac{3}{2 u_{p}}-\frac{1}{2 v_{p}} .
\end{aligned}
$$

Finally, we can transform back to position space to get

$$
\begin{aligned}
\left\langle T_{\mu \nu} j_{\tilde{0}} j_{\tilde{0}} j_{\tilde{0}}\right\rangle_{c b}= & p \times\left(g_{1}(u, v)\left(V(1,2,3)_{\mu} V(1,2,3)_{\nu}-\frac{V(1,2,3)^{2}}{3} \eta_{\mu \nu}\right)\right. \\
& +g_{2}(u, v)\left(V(1,2,3)_{(\mu} V(1,3,4)_{\nu)}-\frac{V(1,2,3) \cdot V(1,3,4)}{3} \eta_{\mu \nu}\right) \\
& \left.+g_{3}(u, v)\left(V(1,3,4)_{\mu} V(1,3,4)_{\nu}-\frac{V(1,3,4)^{2}}{3} \eta_{\mu \nu}\right)\right),
\end{aligned}
$$

where $p=\frac{1}{\left(x_{12} x_{13} x_{14}\right)^{\frac{10}{3}}\left(x_{23} x_{24} x_{34}\right)^{\frac{1}{3}}}, V(i ; j, k)=\frac{x_{i j}^{2}\left(x_{i k}\right)_{\mu}-x_{i k}^{2}\left(x_{i j}\right)_{\mu}}{x_{j k}^{2}}$ and

$$
\begin{aligned}
& g_{1}(u, v)=\frac{u^{2 / 3} v^{2 / 3}}{4 \pi^{3}}-\frac{v^{2 / 3}}{4 \pi^{3} u^{4 / 3}}+\frac{v^{5 / 3}}{2 \pi^{3} u^{4 / 3}}-\frac{v^{8 / 3}}{4 \pi^{3} u^{4 / 3}}+\frac{v^{2 / 3}}{2 \pi^{3} \sqrt[3]{u}}+\frac{v^{5 / 3}}{2 \pi^{3} \sqrt[3]{u}} \\
& g_{2}(u, v)=\frac{u^{2 / 3} v^{2 / 3}}{2 \pi^{3}}+\frac{u^{2 / 3}}{2 \pi^{3} \sqrt[3]{v}}+\frac{u^{5 / 3}}{4 \pi^{3} \sqrt[3]{v}}+\frac{v^{2 / 3}}{2 \pi^{3} \sqrt[3]{u}}+\frac{v^{5 / 3}}{4 \pi^{3} \sqrt[3]{u}}-\frac{3}{4 \pi^{3} \sqrt[3]{u} \sqrt[3]{v}}, \\
& g_{3}(u, v)=\frac{u^{2 / 3} v^{2 / 3}}{4 \pi^{3}}-\frac{u^{2 / 3}}{4 \pi^{3} v^{4 / 3}}+\frac{u^{5 / 3}}{2 \pi^{3} v^{4 / 3}}-\frac{u^{8 / 3}}{4 \pi^{3} v^{4 / 3}}+\frac{u^{2 / 3}}{2 \pi^{3} \sqrt[3]{v}}+\frac{u^{5 / 3}}{2 \pi^{3} \sqrt[3]{v}}
\end{aligned}
$$

The result agrees with [11]. For correlators of type $\left\langle j_{s} j_{\tilde{0}} j_{\tilde{0}} j_{\tilde{0}}\right\rangle$ with $s \geq 4$, the mixed Fourier transform is not so simple, so in practice it was not useful.

\section{Miscellaneous formulas}

In this appendix we write some formulas we used in the text. The nonzero coefficients in equation (2.11) are

$$
\begin{aligned}
a_{1,-1,-1}= & -\left(\gamma_{14}-1\right)\left(2 \gamma_{14}^{2}-\gamma_{14}(4 k+5)+2 k^{2}+5 k+2\right)\left(k^{2}-2 k s-k+s^{2}+s\right), \\
a_{0,0,0}= & -\frac{1}{2}\left(2 \gamma_{14}^{2}-\gamma_{14}(4 k+5)+2 k^{2}+5 k+2\right) \\
& \times\left(-2 \gamma_{12}(k+s)+\gamma_{14}(2 k-2 s+1)+s(2 s+1)\right)(k-s), \\
a_{1,-1,0}= & -\frac{1}{2}\left(2 \gamma_{12}^{2}+\gamma_{12}(4 k-4 s-1)+2 k^{2}-k(4 s+1)+2 s^{2}+s-1\right)\left(\gamma_{14}-1\right) \\
& \times\left(2 k^{2}-4 k s+k+s(2 s-1)\right), \\
a_{0,-1,0}= & \frac{1}{2}\left(\gamma_{14}-1\right)\left(2 k^{2}-4 k s+k+s(2 s-1)\right) \\
& \times\left(2 \gamma_{12}^{2}+\gamma_{12}\left(4 \gamma_{14}-4 s-3\right)+2 \gamma_{14}^{2}-\gamma_{14}(4 s+3)+s(2 s+3)\right),
\end{aligned}
$$




$$
\begin{aligned}
a_{-1,0,1}= & -\frac{1}{2}\left(\gamma_{12}-1\right)\left(2 k^{2}+3 k+1\right) \\
& \times\left(2 \gamma_{12}^{2}+\gamma_{12}\left(4 \gamma_{14}-4 s-3\right)+2 \gamma_{14}^{2}-\gamma_{14}(4 s+3)+s(2 s+3)\right), \\
a_{-1,1,1}= & \frac{1}{2}\left(\gamma_{12}-1\right)\left(2 k^{2}+3 k+1\right)\left(2 \gamma_{14}^{2}-\gamma_{14}(4 k+5)+2 k^{2}+5 k+2\right), \\
a_{0,0,1}= & \frac{1}{2}(k+1)\left(2 \gamma_{12}^{2}+\gamma_{12}(4 k-4 s-1)+2 k^{2}-k(4 s+1)+2 s^{2}+s-1\right) \\
& \times\left(2 \gamma_{12} k+\gamma_{12}-2 \gamma_{14}(k-2 s+1)-s(2 s+1)\right), \\
a_{-1,1,2}= & \left(\gamma_{12}-1\right)\left(k^{2}+3 k+2\right) \\
& \times\left(2 \gamma_{12}^{2}+\gamma_{12}(4 k-4 s-1)+2 k^{2}-k(4 s+1)+2 s^{2}+s-1\right) .
\end{aligned}
$$

The position space correlator $\left\langle j_{4} j_{\tilde{0}} j_{\tilde{0}} j_{\tilde{0}}\right\rangle_{c b}$ is given by (2.2), with

$$
\begin{aligned}
& (1+\sqrt{u}+\sqrt{v})^{3} \times p \times f_{0}(u, v) \\
& =-\frac{(\sqrt{v}-1)^{2}(\sqrt{v}+1)^{4}}{10 u^{9 / 2} v}+\frac{(17 v-\sqrt{v}+17)(\sqrt{v}+1)^{2}}{60 u^{7 / 2} v} \\
& +\frac{3 v+2 \sqrt{v}+3}{30 u^{5 / 2} v}+\frac{1}{90 u^{3 / 2} v}-\frac{(\sqrt{v}-1)^{2}(\sqrt{v}+1)^{5}}{30 u^{5} v}+\frac{(v+6 \sqrt{v}+1)(\sqrt{v}+1)^{3}}{30 u^{4} v} \\
& +\frac{47 v^{3 / 2}+42 v+42 \sqrt{v}+47}{180 u^{3} v}+\frac{\sqrt{v}+1}{30 u^{2} v}, \\
& (1+\sqrt{u}+\sqrt{v})^{3} \times p \times f_{1}(u, v) \\
& =\frac{7 v+6 \sqrt{v}+11}{45 u^{3 / 2} v^{2}}+\frac{\left(3 v^{3 / 2}-3 v+7 \sqrt{v}-7\right)(\sqrt{v}+1)^{3}}{10 u^{7 / 2} v^{2}} \\
& +\frac{22 v^{3 / 2}+17 v^{2}+32 v+44 \sqrt{v}+17}{30 u^{5 / 2} v^{2}}+\frac{\left(3 v^{3 / 2}-3 v+7 \sqrt{v}-7\right)(\sqrt{v}+1)^{4}}{30 u^{4} v^{2}} \\
& +\frac{\left(47 v^{3 / 2}-7 v+91 \sqrt{v}-35\right)(\sqrt{v}+1)^{2}}{90 u^{3} v^{2}} \\
& +\frac{16 v^{3 / 2}+15 v+27 \sqrt{v}+31}{45 u^{2} v^{2}}+\frac{\sqrt{v}+1}{15 u v^{2}}+\frac{1}{45 \sqrt{u} v^{2}}, \\
& (1+\sqrt{u}+\sqrt{v})^{3} \times p \times f_{2}(u, v) \\
& =\frac{\left(10 v^{2}+28 v-35\right)(\sqrt{v}+1)^{2}}{30 u^{5 / 2} v^{3}} \\
& +\frac{69 v^{3 / 2}+49 v^{2}+172 v+168 \sqrt{v}+49}{90 u^{3 / 2} v^{3}}+\frac{\left(10 v^{2}+28 v-35\right)(\sqrt{v}+1)^{3}}{90 u^{3} v^{3}} \\
& +\frac{172 v^{3 / 2}+46 v^{5 / 2}+78 v^{2}+196 v-21 \sqrt{v}-77}{90 u^{2} v^{3}}+\frac{23 v+30 \sqrt{v}+29}{45 \sqrt{u} v^{3}} \\
& +\frac{\sqrt{u}}{9 v^{3}}+\frac{49 v^{3 / 2}+78 v+114 \sqrt{v}+94}{90 u v^{3}}+\frac{\sqrt{v}+1}{3 v^{3}}, \\
& f_{3}(u, v)=f_{1}(v, u), \quad f_{4}(u, v)=f_{0}(v, u), \\
& \text { where } p=\left|x_{1}-x_{3}\right|^{-18}\left|x_{2}-x_{3}\right|^{7}\left|x_{2}-x_{4}\right|^{-11}\left|x_{3}-x_{4}\right|^{7} \text {. }
\end{aligned}
$$




\section{E Contact interactions for scattering amplitudes}

In section 3 we analyse contact interactions in AdS. We conclude that AdS contact diagrams for $\left\langle j_{s} j_{\tilde{0}} j_{\tilde{0}} j_{\tilde{0}}\right\rangle$ violate the bound on chaos if $s \geq 4$, whereas for $s=2$ there is one parity even and one parity odd contact term that does not violate the bound on chaos.

In this appendix we consider the same problem at the level of 4 dimensional scattering amplitudes. We consider on-shell $2 \rightarrow 2$ scattering amplitudes of the type $\left\langle\Phi_{s} \phi \phi \phi\right\rangle$, where the particle $\Phi_{s}$ at position 1 is massless and has spin $s$ and the three other particles are identical massive scalars $\phi$ of mass $m$. It is expected that there is a bijection between contact terms for $d$-dimensional CFT's and $d+1$ dimensional flat space scattering amplitudes [14].

For scattering amplitudes the analog of the bound on chaos is the statement that the amplitude cannot grow more than quadratically in the Regge limit $[29,30]$. This is the Classical Regge Growth (CRG) conjecture of [30] which states that the S-Matrix of a consistent classical theory never grows faster than $s^{2}$ at fixed $t$ - at all physical values of momenta and for every possible choice of the normalized polarization vector $\zeta_{i}$.

In this appendix we perform some calculations for contact scattering amplitudes that support the results of section 3 . We construct contact scattering amplitudes for $\left\langle\Phi_{2} \phi \phi \phi\right\rangle$ and conclude that there is only one parity even and only one parity odd contact scattering amplitude compatible with the CRG conjecture. ${ }^{8}$

Let us discuss the parity even case. In that case the scattering amplitude $T\left(\zeta, p_{1}, p_{2}, p_{3}\right)$ is a function of the polarization $\zeta$ and the momenta. Notice we used momentum conservation to eliminate $p_{4}$. Furthermore the polarization obeys $\zeta \cdot \zeta=p_{1} \cdot \zeta=0$. Thus we have

$$
T\left(\zeta, p_{1}, p_{2}, p_{3}\right)=\left(\zeta \cdot p_{2}\right)^{2} f_{1}(s, t)+\left(\zeta \cdot p_{3}\right)^{2} f_{2}(s, t)+\left(\zeta \cdot p_{2}\right)\left(\zeta \cdot p_{3}\right) f_{3}(s, t),
$$

where we define the Mandelstam invariants as

$$
s=-\left(p_{1}+p_{2}\right)^{2}, \quad t=-\left(p_{1}+p_{3}\right)^{2}, \quad u=-\left(p_{1}+p_{4}\right)^{2} .
$$

The amplitude is constrained by crossing symmetry and gauge invariance. Gauge invariance is the statement that the amplitude is invariant under $\zeta \rightarrow \zeta+\lambda_{1} p_{1}$, where $\lambda_{1}$ is an arbitrary real number. Crossing symmetry and gauge invariance lead to the constraints

$$
\begin{aligned}
f_{2}(s, t) & =\frac{\left(m^{2}-s\right)^{2}}{\left(m^{2}-t\right)^{2}} f_{1}(s, t), & f_{3}(s, t) & =\frac{2\left(s-m^{2}\right)}{\left(m^{2}-t\right)} f_{1}(s, t) \\
f_{1}(s, t) & =\frac{\left(m^{2}-t\right)^{2}}{\left(m^{2}-s\right)^{2}} f_{1}(t, s), & f_{1}(s, t) & =f_{1}(u, t) .
\end{aligned}
$$

We solved the above expressions using polynomials $f_{i}(s, t)=\sum c_{n_{1}, n_{2}, n_{3}}^{i} s^{n_{1}} t^{n_{2}} m^{n_{3}}$. The solution with polynomials of lowest degree is

$$
T=\left(\zeta \cdot p_{2}\right)^{2}\left(p_{1} \cdot p_{3}\right)^{2}+\left(\zeta \cdot p_{3}\right)^{2}\left(p_{1} \cdot p_{2}\right)^{2}-2\left(\zeta \cdot p_{2}\right)\left(\zeta \cdot p_{3}\right)\left(p_{1} \cdot p_{2}\right)\left(p_{1} \cdot p_{3}\right) .
$$

\footnotetext{
${ }^{8}$ A similar analysis for the case of four scalars was done in [10]. There it was found that there are three contact diagrams that do not violate the Regge bound, whose scattering amplitudes are given by $1, s \times t \times u$ and $s^{2}+t^{2}+u^{2}$ respectively.
} 
Let us analyse the Regge limit of this expression in light of the CRG conjecture. We need to parametrize $\zeta$, which we do in the following manner [30]

$$
\zeta=\zeta^{\perp}+\zeta^{\|}, \quad \zeta^{\|}=\alpha_{1} \sqrt{\frac{s t}{u}}\left(\frac{p_{2}}{s-m^{2}}-\frac{p_{3}}{t-m^{2}}\right)+a_{1} p_{1}
$$

$\zeta^{\perp}$ is the component of $\zeta$ that is perpendicular to the plane generated by $p_{1}, p_{2}$ and $p_{3}$, $\zeta^{\|}$is the parallel component. Let us explain the logic for this parametrization. A priori, $\zeta$ depends on 4 independent parameters, but due to the conditions $\zeta^{2}=\zeta \cdot p_{1}=0$ it only depends on two, which we call $\alpha_{1}$ and $a_{1}$. However, the component $a_{1}$ is not physical due to the gauge symmetry of the S-Matrix.

We can now take the expression (E.6) for $\zeta$, plug it into (E.5) and consider the limit $s \rightarrow \infty$ with $t$ fixed. We find that the amplitude grows like $s^{2}$. Thus, it obeys the CRG conjecture.

A similar analysis can be performed for the parity odd contact term. The term that is Regge bounded is

$$
T=\epsilon_{\mu_{1} \mu_{2} \mu_{3} \mu_{4}} \zeta^{\mu_{1}} p_{1}^{\mu_{2}} p_{2}^{\mu_{3}} p_{3}^{\mu_{4}}\left(\left(p_{1} \cdot p_{3}\right)\left(\zeta \cdot p_{2}\right)-\left(p_{1} \cdot p_{2}\right)\left(\zeta \cdot p_{3}\right)\right) .
$$

Open Access. This article is distributed under the terms of the Creative Commons Attribution License (CC-BY 4.0), which permits any use, distribution and reproduction in any medium, provided the original author(s) and source are credited.

\section{References}

[1] O. Aharony, S.M. Chester and E.Y. Urbach, A Derivation of AdS/CFT for Vector Models, JHEP 03 (2021) 208 [arXiv: : 2011.06328] [INSPIRE].

[2] R. de Mello Koch, A. Jevicki, K. Jin and J.P. Rodrigues, $A d S_{4} / C F T_{3}$ Construction from Collective Fields, Phys. Rev. D 83 (2011) 025006 [arXiv:1008.0633] [InSPIRE].

[3] R. de Mello Koch, A. Jevicki, J.P. Rodrigues and J. Yoon, Canonical Formulation of $O(N)$ Vector/Higher Spin Correspondence, J. Phys. A 48 (2015) 105403 [arXiv:1408.4800] [INSPIRE].

[4] R. de Mello Koch, A. Jevicki, K. Suzuki and J. Yoon, AdS Maps and Diagrams of Bi-local Holography, JHEP 03 (2019) 133 [arXiv:1810.02332] [INSPIRE].

[5] J. Maldacena and A. Zhiboedov, Constraining conformal field theories with a slightly broken higher spin symmetry, Class. Quant. Grav. 30 (2013) 104003 [arXiv:1204.3882] [INSPIRE].

[6] S. Jain, V. Malvimat, A. Mehta, S. Prakash and N. Sudhir, The Anomalous Dimension of the Scalar Primary in Chern Simons Vector Models, Phys. Rev. D 101 (2020) 126017 [arXiv: 1906.06342] [INSPIRE].

[7] S. Giombi, V. Gurucharan, V. Kirilin, S. Prakash and E. Skvortsov, On the Higher-Spin Spectrum in Large N Chern-Simons Vector Models, JHEP 01 (2017) 058 [arXiv: 1610.08472] [INSPIRE].

[8] S. Giombi, V. Kirilin and E. Skvortsov, Notes on Spinning Operators in Fermionic CFT, JHEP 05 (2017) 041 [arXiv:1701.06997] [INSPIRE].

[9] E. Skvortsov, Light-Front Bootstrap for Chern-Simons Matter Theories, JHEP 06 (2019) 058 [arXiv: 1811.12333] [INSPIRE]. 
[10] G.J. Turiaci and A. Zhiboedov, Veneziano Amplitude of Vasiliev Theory, JHEP 10 (2018) 034 [arXiv: 1802.04390] [INSPIRE].

[11] Z. Li, Bootstrapping conformal four-point correlators with slightly broken higher spin symmetry and 3D bosonization, JHEP 10 (2020) 007 [arXiv:1906.05834] [INSPIRE].

[12] D.J. Binder, S.M. Chester and M. Jerdee, ABJ Correlators with Weakly Broken Higher Spin Symmetry, arXiv:2103.01969 [INSPIRE].

[13] V.E. Didenko, J. Mei and E.D. Skvortsov, Exact higher-spin symmetry in CFT: free fermion correlators from Vasiliev Theory, Phys. Rev. D 88 (2013) 046011 [arXiv:1301.4166] [INSPIRE].

[14] M.S. Costa, J. Penedones, D. Poland and S. Rychkov, Spinning Conformal Correlators, JHEP 11 (2011) 071 [arXiv:1107.3554] [INSPIRE].

[15] H.-Y. Chen, E.-J. Kuo and H. Kyono, Towards Spinning Mellin Amplitudes, Nucl. Phys. B 931 (2018) 291 [arXiv:1712.07991] [INSPIRE].

[16] V. Gonçalves, J. Penedones and E. Trevisani, Factorization of Mellin amplitudes, JHEP 10 (2015) 040 [arXiv: 1410.4185] [InSPIRE].

[17] J. Faller, S. Sarkar and M. Verma, Mellin Amplitudes for Fermionic Conformal Correlators, JHEP 03 (2018) 106 [arXiv:1711.07929] [INSPIRE].

[18] P. Kravchuk and D. Simmons-Duffin, Counting Conformal Correlators, JHEP 02 (2018) 096 [arXiv: 1612.08987] [INSPIRE].

[19] G.F. Cuomo, D. Karateev and P. Kravchuk, General Bootstrap Equations in 4D CFTs, JHEP 01 (2018) 130 [arXiv:1705.05401] [INSPIRE].

[20] J. Maldacena, D. Simmons-Duffin and A. Zhiboedov, Looking for a bulk point, JHEP 01 (2017) 013 [arXiv: 1509.03612] [INSPIRE].

[21] J. Penedones, J.A. Silva and A. Zhiboedov, Nonperturbative Mellin Amplitudes: Existence, Properties, Applications, JHEP 08 (2020) 031 [arXiv: 1912.11100] [INSPIRE].

[22] J. Maldacena, S.H. Shenker and D. Stanford, A bound on chaos, JHEP 08 (2016) 106 [arXiv: 1503.01409] [INSPIRE].

[23] I. Heemskerk, J. Penedones, J. Polchinski and J. Sully, Holography from Conformal Field Theory, JHEP 10 (2009) 079 [arXiv:0907.0151] [INSPIRE].

[24] T. Hartman, S. Kundu and A. Tajdini, Averaged Null Energy Condition from Causality, JHEP 07 (2017) 066 [arXiv: 1610.05308] [INSPIRE].

[25] M.S. Costa, V. Goncalves and J. Penedones, Conformal Regge theory, JHEP 12 (2012) 091 [arXiv:1209.4355] [INSPIRE].

[26] M.S. Costa, V. Gonçalves and J. Penedones, Spinning AdS Propagators, JHEP 09 (2014) 064 [arXiv: 1404.5625] [INSPIRE].

[27] S. Caron-Huot and Y.-Z. Li, Helicity basis for three-dimensional conformal field theory, arXiv: 2102.08160 [INSPIRE].

[28] S. Jain, R.R. John and V. Malvimat, Constraining momentum space correlators using slightly broken higher spin symmetry, JHEP 04 (2021) 231 [arXiv:2008.08610] [INSPIRE].

[29] S. Caron-Huot, D. Mazac, L. Rastelli and D. Simmons-Duffin, Sharp Boundaries for the Swampland, arXiv:2102.08951 [INSPIRE]

[30] S.D. Chowdhury, A. Gadde, T. Gopalka, I. Halder, L. Janagal and S. Minwalla, Classifying and constraining local four photon and four graviton S-matrices, JHEP 02 (2020) 114 [arXiv: 1910.14392] [INSPIRE]. 